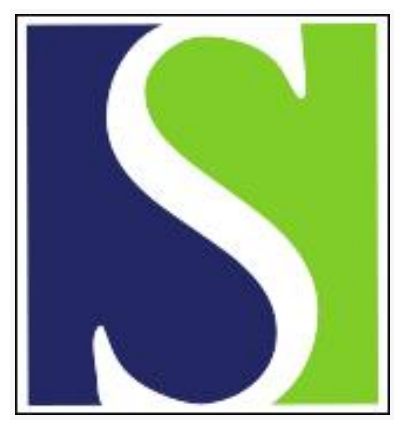

Scand J Work Environ Health 2008;34(1):5-22

https://doi.org/10.5271/sjweh.1194

Issue date: 29 Feb 2008

Nightshift work and risk of breast cancer and other cancers-a critical review of the epidemiologic evidence

by Kolstad HA

Affiliation: Aarhus University Hospital, Department of Occupational Medicine, Norrebrogade 44, DK-8000 Århus C, Denmark. hkols@as.aaa.dk

Refers to the following texts of the Journal: 2007;33(1):66-73 1999;25 suppl 2:1-116 2006;32(6):502-514 1999;25(2):85-99 2007;33(5):336-343

The following articles refer to this text: $2010 ; 36(2): 134-141$; 2010;36(2):163-179; 2010;36(2):81-84; 2010;36(6):515-516; 2011;37(4):263-275; 2011;37(6):533-538; 2012;38(6):590-599; 2012;38(6):553-559; 2013;39(4):425-426; 2013;39(5):431-447; 2014;40(5):502-510; 2015;41(3):268-279; 2017;43(6):560-568; 2019;45(5):458-464

Key terms: breast cancer; cancer; chronobiology disorder; circadian rhythm; epidemiologic evidence; nightshift work; occupational exposure; occupational health; review; risk; shift work

This article in PubMed: www.ncbi.nlm.nih.gov/pubmed/18427694

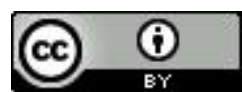




\title{
Nightshift work and risk of breast cancer and other cancers-a critical review of the epidemiologic evidence
}

\author{
by Henrik A Kolstad, MD ${ }^{1}$
}

\begin{abstract}
Kolstad HA. Nightshift work and risk of breast cancer and other cancers-a critical review of the epidemiologic evidence. Scand J Work Environ Health. 2008;34(1):5-22.
\end{abstract}

\begin{abstract}
Objectives This systematic review concerns the role of nightshift work in the risk of breast cancer or other cancers.

Methods Studies that specifically included information on nightshift or shift work and reported cancer occurrence were focused upon. A systematic search of Medline and the Science Citation Index was conducted until May 2007. The quality of each paper was discussed with respect to design, exposure and outcome information, bias, confounding, and exposure-response assessment.

Results Thirteen relevant reports were found, and eight reported the relative risk for breast cancer, three for prostate cancer, three for colon cancer, and four for all cancers. Most of the studies had crude information about nightshift work, four register-linked studies had no individual exposure information but relied on exposure probabilities assessed on a group level, and no studies analyzed cancer risk according to the cumulative number of night shifts (however, most of the studies did so according to the number of years of nightshift work). Confounding did not seem to be of major concern. The presentation of the results was not always complete, and it would have been appreciated if the reasons for leaving some findings out had been reported. There were indications of a long-term effect of nightshift work (more than 20-30 years), but the number of positive studies was small. In addition, they were all conducted among nurses, and the risk estimates were only moderately increased. This situation makes the results sensitive to bias, chance, and confounding.

Conclusions There is limited evidence for a causal association between nightshift work and breast cancer, while there is insufficient evidence for prostate cancer, colon cancer, and overall cancer.
\end{abstract}

Key terms chronobiology disorder; circadian rhythm; occupational exposure; occupational health; shift work.

In 1987, it was hypothesized that the rising risk of breast cancer seen in industrialized societies was, at least partly, due to the increased use of electric lighting at night (1). It was suggested that light at night could suppress melatonin output and increase estrogen levels and thereby increase the risk of breast cancer. The idea was based on experiments with rodents, which showed that constant light affected mammary tumorigenesis, and epidemiologic results that breast cancer risk was highest in the most-industrialized societies. The melatonin hypothesis stimulated various lines of research, from laboratory studies using animal models to epidemiologic studies of humans exposed to light at night and nightshift work. Recently, the hypothesis has evolved from merely a question of suppression of melatonin output to a question of disruption of the circadian rhythm, interaction with clock genes, and light exposures in early life (2).
Megdal et al (3) reviewed the epidemiologic evidence of nightshift work and breast cancer in 2005. My review is focused on prostate, colon, and all cancers, in addition to breast cancer. It includes six studies not included in the earlier review (4-9), and it leaves out seven studies of flight attendants that provided no information about nightshift work.

Altogether 429900 European women were diagnosed with breast cancer in 2006, and breast cancer has become the most common form of cancer in Europe (13.5\% of all cancer cases) (10). Fifty percent of all breast cancer cases can be attributed to known risk factors (11), primarily prolonged exposure to endogenous and exogenous female sex hormones (12-15), but also alcohol (16), adiposity, and excessive weight gain (17).

About $20 \%$ of employees or self-employed workers in the countries in the European Union (EU) work at least one night a month (at least 2 hours between 2200

1 Aarhus University Hospital, Department of Occupational Medicine, Århus, Denmark.

Correspondence to: Dr Henrik Kolstad, Aarhus University Hospital, Department of Occupational Medicine, Norrebrogade 44, DK-8000 Århus C, Denmark. [E-mail: hkols@as.aaa.dk] 
and 0500) (18). Ten percent work 1-5 nights, and 10\% work more than 5 nights per month. Furthermore, $0.4 \%$ work permanent night shifts (19). Night work is the most prevalent $(>30 \%)$ in agriculture, hotels, restaurants, transport, communication, and health (18).

\section{Exposures of nightshift work}

\section{Light}

In offices and hospitals, the illuminance at the cornea is about 100-300 lux during night and daytime work compared with 10000 lux outdoors during the day and 0.1-5 lux when asleep during the night and day (20). A threshold level of 30 lux of white light has been suggested for melatonin suppression (20).

\section{Behavioral factors}

A previous review reported that 9 out of 11 studies showed 1.07-1.48 times more smokers among shift workers than among day workers, while two studies showed reduced prevalence ratios (0.56 and 0.96) (21). That review documented no strong indications that alcohol consumption or exercise differ between shift workers and day workers. Only few differences were observed with respect to nutritional intake. However, the frequency and timing of meals may change during shift work, and there were some indications that shift workers are heavier than day workers ( 2 of 10 studies; 8 showed no difference).

The distribution of behavioral factors among nightshift and day workers was reported in five of the studies included in this review $(6,7,22-25)$. Table 1 presents an overview of this information and shows that nightshift workers more often smoke (an average of about 20-30\% more smokers), have a higher body mass index (BMI), and less often use hormone replacement therapy than day workers. Nightshift workers also seem to have early menarche more often, but no consistent patterns were apparent for other reproductive factors across the five studies. It should, however, be noted that a 3-6 times higher alcohol consumption was reported for nightshift workers than for day workers in one study (25).

Table 1. Characteristics of nightshift workers relative to day workers. Results of five epidemiologic studies in 2001-2007. ( $F=$ female, $M$ $=$ male,$\uparrow=$ characteristic more prevalent or more pronounced among nightshift workers than among daytime workers, $\downarrow=$ characteristic less prevalent or less pronounced among nightshift workers than among daytime workers, $0=$ no substantial difference between nightshift and daytime workers, $\mathrm{BMI}=$ body mass index, $\mathrm{HRT}$ = hormone replacement therapy)

\begin{tabular}{|c|c|c|c|c|c|}
\hline \multirow[t]{2}{*}{ Characteristic } & \multicolumn{5}{|c|}{ Prevalence or magnitude of characteristic among nightshift workers relative to daytime workers by study a } \\
\hline & $\begin{array}{c}\text { Schernhammer et al, } \\
2001 \text { (22); Schern- } \\
\text { hammer et al, } 2003 \text { (23) }\end{array}$ & $\begin{array}{l}\text { 0'Leary et al, } \\
2006(6)\end{array}$ & $\begin{array}{l}\text { Schernhammer } \\
\text { et al, } 2006(24)\end{array}$ & Kubo et al, $2006(7)$ & Hansen, 2001 (25) \\
\hline Gender & $\mathrm{F}$ & $\mathrm{F}$ & $\mathrm{F}$ & $M$ & $\mathrm{~F}$ \\
\hline Age & $\uparrow$ & $\downarrow$ & $\uparrow$ & .. & .. \\
\hline Early menarche & $\begin{array}{c}\uparrow \\
\text { (Age at menarche }<12 \text { years: } \\
26.3 \% \text { versus } 21.8 \% \text { ) }\end{array}$ & .. & $\begin{array}{c}\uparrow \\
\text { (Age at menarche }<14 \text { years: } \\
\text { 86\% versus } 83 \% \text { ) }\end{array}$ & .. &.. \\
\hline Nulliparous & 0 & $\downarrow$ & .. &.$\cdot$ & .. \\
\hline Postmenopausal & $\uparrow$ & $\downarrow$ & $\uparrow$ & .. & .. \\
\hline Young at first birth & $\downarrow$ & .. & $\uparrow$ & .. & .. \\
\hline Body mass index & $\begin{array}{c}\uparrow \\
\left(\mathrm{BMI}>25 \mathrm{~kg} / \mathrm{m}^{2}:\right. \\
42.7 \% \text { versus } 40.1 \%)\end{array}$ & .. & $\begin{array}{c}\uparrow \\
\left(\mathrm{BMI}, \mathrm{kg} / \mathrm{m}^{2}:\right. \\
28.9 \text { versus 25.4) }\end{array}$ & $\begin{array}{c}\uparrow \\
\left(\mathrm{BMI}>23.9 \mathrm{~kg} / \mathrm{m}^{2}:\right. \\
34.4 \% \text { versus } 30.8 \%)\end{array}$ & .. \\
\hline Family history & 0 & $\downarrow$ & 0 & $\uparrow$ & .. \\
\hline Benign breast disease & $\downarrow$ & $\uparrow$ & $\uparrow$ & .. & .. \\
\hline Oral contraceptive & $\downarrow$ & $\uparrow$ & 0 & .. & .. \\
\hline $\begin{array}{l}\text { Hormone replacement } \\
\text { therapy }\end{array}$ & $\begin{array}{c}\downarrow \\
\text { (Current HRT } \geq \text { years: } \\
9.1 \% \text { versus } 10.9 \% \text { ) }\end{array}$ & $\begin{array}{c}\downarrow \\
\text { (Ever HRT: } \\
\text { 24.0\% versus 34.4\%) }\end{array}$ & .. & .. & .. \\
\hline Physical activity & .. & .. & .. & $\downarrow$ & .. \\
\hline Alcohol & $\downarrow$ & $\uparrow$ & $\downarrow$ & 0 & $\uparrow$ \\
\hline Smoking & $\begin{array}{l}\uparrow \\
\text { (Current or former smoker: } \\
24.6 \% \text { versus } 17.4 \% \text { ) }\end{array}$ & $\begin{array}{c}\uparrow \\
\text { (Current smokers: } \\
14 \% \text { versus } 10 \% \text { ) }\end{array}$ & $\begin{array}{l}\uparrow \\
\text { (Current or former smoker: } \\
78.2 \% \text { versus } 76.5 \% \text { ) }\end{array}$ & & \\
\hline Socioeconomic status & $\downarrow$ & 0 & $\cdot \cdot$ &.$\cdot$ & .. \\
\hline Indoor work & .. & .. & .. & $\uparrow$ &.. \\
\hline
\end{tabular}

a If consistent across the studies, detailed results are presented in parentheses. 


\section{Sleep deprivation and stress}

Nightshift workers often complain about insufficient sleep because the quality of daytime sleep may be worse than nighttime sleep $(20,26)$. Activation of the hypothalamic-pituitary-adrenal (HPA) axis has been suggested as a major mediator of illness and disease (eg, cardiovascular disease) among shift workers (27), and shift work is related to lower job control, but no difference in work demands (28).

\section{Melatonin in nightshift workers}

A large field study of nurses recently showed a lower concentration of urinary 6-sulfatoxy melatonin (the major metabolite in urine) in mixed shift workers during their nightshifts than during their days off (intra-individual comparisons) and also a lower concentration than that of fixed dayshift workers (interindividual comparisons) (29). Urinary 6-sulfatoxy melatonin levels were specified for 3-hour intervals during 24-hour periods. Fixed nightshift workers showed somewhat lower concentrations, of borderline statistical significance, than mixed nightshift workers during nightshift workdays. Twenty-four-hour 6-sulfatoxy melatonin output was not reported, but it showed no significant association with nightshift work (personal communication, Åse Marie Hansen). Borugian et al (30) found lower 24-hour melatonin levels among rotating shift workers during night shifts but higher levels during day shifts or days off than for day workers. Shernhammer et al (31) showed lowered 6-sulfatoxy melatonin levels in the morning urine of 14 nurses that worked at least one night shift during the previous 2 weeks than for nurses working no night shifts during this period.

\section{Methods}

Three methods were used in combination to identify the epidemiologic literature relevant to nightshift work and cancer risk. First, a computerized search was conducted in PubMed in May 2007. Search terms included night work, night shift, or shift work (text words) or circadian rhythm, work schedule tolerance, circadian disruption and chronobiology disorders (MeSH terms or subheadings) and cancer (text term) or neoplasms and risk, rate, odds ratio, incidence, or mortality (MeSH terms). Then references were extracted from the bibliographies of the articles identified. All of the abstracts were reviewed, and the final set of studies was decided upon. I included original epidemiologic studies that specifically had information on night or shift work and the risk of cancer. No animal studies were included. Finally, I conducted a search in Science Citation Index that was based on three core publications $(22,25,32)$.

Altogether 426 articles were identified. After the abstracts were reviewed, 61 articles were selected for a detailed evaluation. Of these, 12 were original epidemiologic studies of night or shift workers and cancer risk

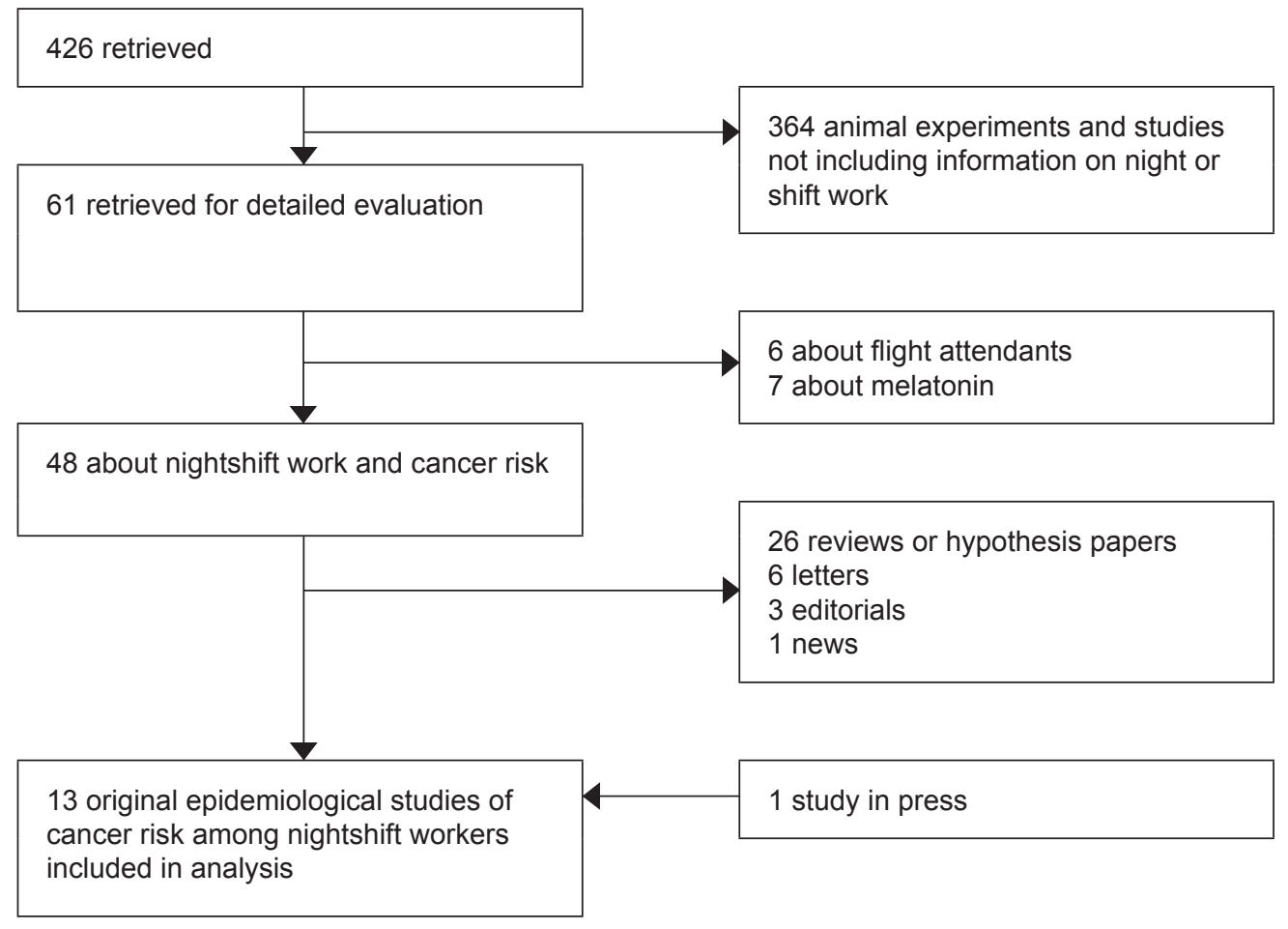

Figure 1. Flow chartnightshift work and risk of cancer. 
and thus met the inclusion criteria $(4-8,22-25,32-34)$ (figure 1). I also identified 26 reviews or hypothesis papers $(2,3,20,35-57), 6$ letters (58-63), 3 editorials (64-66), and 1 news report (67) in addition to 6 papers focusing on flight attendants (68-73) and 7 focusing specifically on melatonin (74-79). Finally, I included the study by Schwartzbaum et al that was accepted for publication but not yet published in the Scandinavian Journal of Work Environment \& Health (9).

The 13 selected studies included 8 studies on breast cancer $(6,9,22,24,25,32-34), 3$ studies on prostate cancer (7-9), 3 studies on colon cancer $(9,23,33)$, and 4 studies on all cancer $(4,5,9,33)$. Tynes et al (33) reported the risk of breast cancer, colon cancer, and all cancer, and Schwartzbaum et al (9) reported the risk of breast cancer, prostate cancer, colon cancer, and all cancer. Tynes et al (33) and Schwartzbaum et al (9) reported on other major cancer sites as well, but I only reviewed sites studied in at least three studies. The 2001 study about breast cancer and the 2003 study about colon cancer by Schernhammer et al were based on the same study population $(22,23)$, which did not overlap with the
2006 study about breast cancer (24). Table 2 presents the main characteristics of the 13 studies, and table 3 presents the principal results, cancer site by cancer site.

Epidemiologic studies of pilots, flight attendants, physicians, radiologists, military and police employees, fire fighters, or police or law enforcement personnel that have been included in previous reviews were not included $(45,52)$, since these studies did not specifically include any information about nightshift work for the study participants. The former review authored by Megdal and her colleagues (3) focused on cancer risk among aircrew, and I therefore included studies of airline flight attendants and pilots, in addition to other relevant toxicologic and epidemiologic data in the section with contributory evidence. That part of the review is not comprehensive or systematic; instead the intention was to include a balanced set of the most relevant studies.

Finally, the degree of evidence for causal associations between nightshift work and each of the reviewed cancer sites was rated according to the criteria of the Scientific Committee of the Danish Society of Occupational

Table 2. Main characteristics of 13 epidemiologic studies of nightshift work and risk of cancer, 1972-2007. (ICD-0 = International Classification of Diseases for Oncology; ICD-7 = International Classification of Diseases, 7th revision; BMI = body mass index)

\begin{tabular}{|c|c|c|c|c|c|c|c|}
\hline Study & $\begin{array}{l}\text { Cancer } \\
\text { sites } \\
\text { included } \\
\text { in review }\end{array}$ & $\begin{array}{l}\text { Design, study population, } \\
\text { study period and number of } \\
\text { participants (participation } \\
\text { rate) }\end{array}$ & $\begin{array}{l}\text { Source of } \\
\text { exposure } \\
\text { infor- } \\
\text { mation }\end{array}$ & $\begin{array}{l}\text { Measure of exposure } \\
\text { (prevalence in study base) and } \\
\text { exposure period }\end{array}$ & $\begin{array}{l}\text { Source of outcome } \\
\text { information } \\
\text { and latency or } \\
\text { induction period }\end{array}$ & Controlled covariates & $\begin{array}{l}\text { Exposure- } \\
\text { response } \\
\text { assessment }\end{array}$ \\
\hline $\begin{array}{l}\text { Tynes et } \\
\text { al, } 1996 \\
\text { (33); } \\
\text { Norway }\end{array}$ & $\begin{array}{l}\text { Breast, } \\
\text { colon, all } \\
\text { cancer }\end{array}$ & $\begin{array}{l}\text { Nested case-control regis- } \\
\text { ter-linked study within co- } \\
\text { hort of telegraph operators, } \\
1961-1991 ; 50 \text { breast can- } \\
\text { cer cases, } 259 \text { age-matched } \\
\text { controls, } 140 \text { all cancers } \\
(98 \%)\end{array}$ & $\begin{array}{l}\text { Journa- } \\
\text { list- and } \\
\text { research- } \\
\text { er-classi- } \\
\text { fied ships } \\
\text { according } \\
\text { to extent } \\
\text { of shift } \\
\text { work }\end{array}$ & $\begin{array}{l}\text { Four categories (0-3) reflecting } \\
\text { presence in radio room during } \\
\text { day and night (83-90\%); total } \\
\text { work history since certification }\end{array}$ & $\begin{array}{l}\text { National cancer } \\
\text { register; separate } \\
\text { analyses for expo- } \\
\text { sures prior to } 30 \\
\text { years of age and } \\
\text { risk before or after } \\
50 \text { years of age }\end{array}$ & $\begin{array}{l}\text { Duration of employ- } \\
\text { ment and age at first } \\
\text { birth ( } 3 \text { levels) for } \\
\text { women born } \geq 1935\end{array}$ & $\begin{array}{l}\text { Duration } \\
\text { (years) and } \\
\text { shiftwork } \\
\text { category } \\
\text { × duration; } \\
\text { test for trend } \\
\text { of continuous } \\
\text { term }\end{array}$ \\
\hline $\begin{array}{l}\text { Davis et } \\
\text { al, } 2001 \\
\text { (32); } \\
\text { United } \\
\text { States }\end{array}$ & Breast & $\begin{array}{l}\text { Population-based case-con- } \\
\text { trol study, } 1992-1995 ; \\
767 \text { cases }(78 \%) \text { and } \\
743 \text { controls }(75 \%)\end{array}$ & $\begin{array}{l}\text { Ques- } \\
\text { tionnaire } \\
\text { at diagno- } \\
\text { sis or on } \\
\text { reference } \\
\text { date }\end{array}$ & $\begin{array}{l}\text { Graveyard shift: beginning } \\
\text { after } 1900 \text { and ending before } \\
0900(3-5 \%) ; 10 \text { years prior } \\
\text { to diagnosis or reference date; } \\
\text { information on graveyard shifts } \\
\text { during total work history was } \\
\text { obtained but no risk estimates } \\
\text { reported }\end{array}$ & $\begin{array}{l}\text { Regional cancer } \\
\text { register; no analy- } \\
\text { ses by latency }\end{array}$ & $\begin{array}{l}\text { Age, parity, family his- } \\
\text { tory, oral contracep- } \\
\text { tives, and hormone- } \\
\text { replacement therapy }\end{array}$ & $\begin{array}{l}\text { Hours/week } \\
\text { and duration } \\
\text { (years); test } \\
\text { for trend of } \\
\text { continuous } \\
\text { term }\end{array}$ \\
\hline $\begin{array}{l}\text { Schern- } \\
\text { hammer } \\
\text { et al, } \\
2001 \\
\text { (22); } \\
\text { United } \\
\text { States }\end{array}$ & Breast & $\begin{array}{l}\text { Prospective cohort of } \\
\text { nurses, 1988-1998; } \\
78 \text { 562; } 2441 \text { cases }(82 \%)\end{array}$ & $\begin{array}{l}\text { Ques- } \\
\text { tion- } \\
\text { naire at } \\
\text { baseline } \\
\text { (1988) }\end{array}$ & $\begin{array}{l}\text { Rotating night shifts; at least } \\
3 \text { nights/month, in addition to } \\
\text { days or evenings in that month } \\
(60 \%) \text {; total work history prior } \\
\text { to baseline }\end{array}$ & $\begin{array}{l}\text { Self-reports con- } \\
\text { firmed by medical } \\
\text { records or national } \\
\text { death index; pa- } \\
\text { thology reports for } \\
93 \% \text {; no analyses } \\
\text { by latency }\end{array}$ & $\begin{array}{l}\text { Age, age at menarche, } \\
\text { parity, age at first } \\
\text { birth, weight change, } \\
\text { BMI, family history, } \\
\text { benign breast disease, } \\
\text { oral contraceptives, } \\
\text { alcohol, calendar year, } \\
\text { age at menopause, } \\
\text { hormone replacement } \\
\text { therapy, menopausal } \\
\text { status and height }\end{array}$ & $\begin{array}{l}\text { Duration on } \\
\text { rotating night } \\
\text { shifts (years): } \\
\text { never, } \\
1-14,15-29 \text {, } \\
\geq 30 \text {; test } \\
\text { for trend of } \\
\text { continuous } \\
\text { term }\end{array}$ \\
\hline $\begin{array}{l}\text { Hansen, } \\
2001 \\
\text { (25); } \\
\text { Den- } \\
\text { mark }\end{array}$ & Breast & $\begin{array}{l}\text { Population-based register- } \\
\text { linked nested case-control } \\
\text { study, study period not } \\
\text { reported; } 6281 \text { cases ( } 93 \%) \\
\text { and individually matched } \\
6024 \text { controls (participation } \\
\text { rate not reported) }\end{array}$ & $\begin{array}{l}\text { Records } \\
\text { of nation- } \\
\text { al pension } \\
\text { fund }\end{array}$ & $\begin{array}{l}\text { Job exposure matrix classified } \\
4 \text { trades with at least } 60 \% \text { em- } \\
\text { ployees working predominantly } \\
\text { at night }(5 \%) \text {; total work history } \\
\text { since } 1964\end{array}$ & $\begin{array}{l}\text { National cancer } \\
\text { register; 5-year } \\
\text { latency period }\end{array}$ & $\begin{array}{l}\text { Number of children, } \\
\text { age at birth of first } \\
\text { and last child, current } \\
\text { job title }\end{array}$ & $\begin{array}{l}\text { No exposure- } \\
\text { response } \\
\text { assessment; } \\
>6 \text { years of } \\
\text { employment } \\
\text { reported } \\
\text { separately } \\
\quad \text { (continued) }\end{array}$ \\
\hline
\end{tabular}


Table 2. Continued.

\begin{tabular}{|c|c|c|c|c|c|c|c|}
\hline Study & $\begin{array}{l}\text { Cancer } \\
\text { sites } \\
\text { included } \\
\text { in review }\end{array}$ & $\begin{array}{l}\text { Design, study population, } \\
\text { study period and number of } \\
\text { participants (participation } \\
\text { rate) }\end{array}$ & $\begin{array}{l}\text { Source of } \\
\text { exposure } \\
\text { infor- } \\
\text { mation }\end{array}$ & $\begin{array}{l}\text { Measure of exposure } \\
\text { (prevalence in study base) and } \\
\text { exposure period }\end{array}$ & $\begin{array}{l}\text { Source of outcome } \\
\text { information } \\
\text { and latency or } \\
\text { induction period }\end{array}$ & Controlled covariates & $\begin{array}{l}\text { Exposure- } \\
\text { response } \\
\text { assessment }\end{array}$ \\
\hline $\begin{array}{l}\text { Lie et } \\
\text { al, } 2005 \\
(34) ; \\
\text { Norway }\end{array}$ & Breast & $\begin{array}{l}\text { Register-linked case-control } \\
\text { study of nurses, 1960-1982; } \\
537 \text { cases, } 2143 \text { controls } \\
\text { matched by year of birth } \\
\text { (participation rate not re- } \\
\text { ported) }\end{array}$ & $\begin{array}{l}\text { National } \\
\text { register } \\
\text { of nurses } \\
\text { and } \\
\text { national } \\
\text { censuses }\end{array}$ & $\begin{array}{l}\text { Work at infirmaries, except } \\
\text { managerial, teaching, physio- } \\
\text { therapy work or work in outpa- } \\
\text { tient departments (90\%); total } \\
\text { work history }\end{array}$ & $\begin{array}{l}\text { National cancer } \\
\text { register; } 20 \text {-year } \\
\text { latency period }\end{array}$ & $\begin{array}{l}\text { Age at birth of first } \\
\text { child, number of } \\
\text { children, and total } \\
\text { employment time }\end{array}$ & $\begin{array}{l}\text { Duration } \\
\text { (years) of } \\
\text { work in infir- } \\
\text { maries }\end{array}$ \\
\hline $\begin{array}{l}\text { Schern- } \\
\text { hammer } \\
\text { et al, } \\
2006 \\
(24) \text {; } \\
\text { United } \\
\text { States }\end{array}$ & Breast & $\begin{array}{l}\text { Prospective cohort of } \\
\text { nurses, 1989-2001; } \\
115022 ; 1352 \text { cases } \\
(99.5 \%)\end{array}$ & $\begin{array}{l}\text { Ques- } \\
\text { tion- } \\
\text { naire at } \\
\text { baseline } \\
\text { (1989) } \\
\text { and in } \\
1991, \\
1993 \text {, } \\
1997 \text { and } \\
2001\end{array}$ & $\begin{array}{l}\text { Rotating night shifts, at least } \\
3 \text { nights/month, in addition to } \\
\text { days or evenings in that month } \\
(68 \%) \text {; total work history prior } \\
\text { to diagnosis; information on } \\
\text { permanent night shifts were } \\
\text { obtained but not reported }\end{array}$ & $\begin{array}{l}\text { Self-reports } \\
\text { confirmed by } \\
\text { medical records } \\
\text { or national death } \\
\text { index; pathology } \\
\text { reports for } 98 \% \text {; } \\
\text { no analyses by } \\
\text { latency }\end{array}$ & $\begin{array}{l}\text { Age, age at menarche, } \\
\text { menopausal status, } \\
\text { age at menopause, } \\
\text { age at first birth and } \\
\text { parity, BMI, alcohol } \\
\text { consumption, oral con- } \\
\text { traceptives, hormone- } \\
\text { replacement therapy, } \\
\text { smoking, benign breast } \\
\text { disease, family history, } \\
\text { and physical activity }\end{array}$ & $\begin{array}{l}\text { Duration } \\
\text { (years) of } \\
\text { rotating } \\
\text { night shifts: } \\
\text { never, 1-9, } \\
10-19, \geq 20 \text {; } \\
\text { test for } \\
\text { trend of } \\
\text { continuous } \\
\text { term }\end{array}$ \\
\hline $\begin{array}{l}\text { O’Leary } \\
\text { et al, } \\
2006(6) ; \\
\text { United } \\
\text { States }\end{array}$ & Breast & $\begin{array}{l}\text { Population-based case-con- } \\
\text { trol study, 1996-1997; } \\
487 \text { cases (?\%) and } \\
509 \text { controls (?\%) }\end{array}$ & $\begin{array}{l}\text { Interview } \\
\text { at diagno- } \\
\text { sis or on } \\
\text { reference } \\
\text { date }\end{array}$ & $\begin{array}{l}\text { Overnight shifts; starting after } \\
1900 \text { and continuing to the fol- } \\
\text { lowing morning }(10 \%) ; 15 \text { years } \\
\text { prior to diagnosis or reference } \\
\text { date }\end{array}$ & $\begin{array}{l}\text { Weekly hospital } \\
\text { contacts; no } \\
\text { analyses by } \\
\text { latency }\end{array}$ & $\begin{array}{l}\text { Age, parity, family his- } \\
\text { tory, education, benign } \\
\text { breast disease }\end{array}$ & $\begin{array}{l}\text { Duration } \\
\text { (years) of } \\
\text { overnight } \\
\text { shift work }\end{array}$ \\
\hline $\begin{array}{l}\text { Schwartz- } \\
\text { baum } \\
\text { et al, } \\
2007 \text { (9); } \\
\text { Sweden }\end{array}$ & $\begin{array}{l}\text { Breast, } \\
\text { prostate, } \\
\text { colon, all } \\
\text { cancer }\end{array}$ & $\begin{array}{l}\text { Population-based register- } \\
\text { linked cohort study, 1971- } \\
1989 \text {, of gainfully employed } \\
\text { ( } \geq 20 \text { hours/week) in } 1960 \\
\text { and } 1970 ; 69759 \text { men and } \\
3057 \text { women, } 6792 \text { cancer } \\
\text { cases (participation rate not } \\
\text { reported) }\end{array}$ & $\begin{array}{l}\text { Popu- } \\
\text { lation } \\
\text { censuses } \\
\text { in } 1960 \\
\text { and } 1970\end{array}$ & $\begin{array}{l}\text { Job title and industry combina- } \\
\text { tions classified by job-exposure } \\
\text { matrix with at least } 40 \% \text { work- } \\
\text { ing rotating shifts ( } 3 \text { or more } \\
\text { shifts per day) or working any } \\
\text { hour } 0100-0400 \text { at least once a } \\
\text { week ( } 3 \% \text { of men and } 0.3 \% \text { of } \\
\text { women); } 1960-1970\end{array}$ & $\begin{array}{l}\text { National cancer } \\
\text { register; no analy- } \\
\text { ses by latency }\end{array}$ & $\begin{array}{l}\text { Age, socioeconomic } \\
\text { status, occupational } \\
\text { position, county of } \\
\text { residence, marital } \\
\text { status, urbanization }\end{array}$ & $\begin{array}{l}\text { Shift work } \\
\text { in } 1960 \text { and } \\
1970 \text { as } \\
\text { proxy for } \\
\text { duration of } \\
\text { shift work } \\
\text { (>10 years) }\end{array}$ \\
\hline $\begin{array}{l}\text { Kubo et } \\
\text { al, } 2006 \\
\text { (7); } \\
\text { Japan }\end{array}$ & Prostate & $\begin{array}{l}\text { Prospective population- } \\
\text { based cohort study, 1988- } \\
1997 ; 14052 ; 31 \text { cases } \\
(83 \%)\end{array}$ & $\begin{array}{l}\text { Ques- } \\
\text { tionnaire } \\
\text { at base- } \\
\text { line }\end{array}$ & $\begin{array}{l}\text { The longest held work schedule; } \\
\text { fixed night work }(7 \%) \text { and alter- } \\
\text { nate night and day work }(13 \%)\end{array}$ & $\begin{array}{l}\text { Regional cancer } \\
\text { registers }\end{array}$ & $\begin{array}{l}\text { Age, study area, family } \\
\text { history, BMI, smoking, } \\
\text { alcohol, job type, phys- } \\
\text { ical activity, stress, } \\
\text { education, marriage } \\
\text { status }\end{array}$ & $\begin{array}{l}\text { No expo- } \\
\text { sure-re- } \\
\text { sponse } \\
\text { assessment }\end{array}$ \\
\hline $\begin{array}{l}\text { Conlon } \\
\text { et al, } \\
2007 \text { (8); } \\
\text { Canada }\end{array}$ & Prostate & $\begin{array}{l}\text { Population-based case-con- } \\
\text { trol study, 1995-1998; } \\
760 \text { cases, } 1632 \text { age- } \\
\text { matched controls (response } \\
\text { rate not reported) }\end{array}$ & $\begin{array}{l}\text { Ques- } \\
\text { tionnaire } \\
\text { filled out } \\
\text { retrospec- } \\
\text { tively }\end{array}$ & $\begin{array}{l}\text { Full-time rotating shifts }(44 \%) \text {; } \\
\text { total work history; information } \\
\text { on part-time rotating shifts and } \\
\text { evening or night shifts were } \\
\text { obtained but no risk estimates } \\
\text { were reported }\end{array}$ & $\begin{array}{l}\text { Cancer registry; } \\
\text { four-level latency } \\
\text { analysis }\end{array}$ & Age and family history & $\begin{array}{l}\text { Duration } \\
\text { (years) of } \\
\text { full-time } \\
\text { rotating } \\
\text { shifts }\end{array}$ \\
\hline $\begin{array}{l}\text { Schern- } \\
\text { hammer } \\
\text { et al, } \\
2003 \\
(23) ; \\
\text { United } \\
\text { States }\end{array}$ & Colon & $\begin{array}{l}\text { Prospective cohort of } \\
\text { nurses, 1988-1998; } 78586 ; \\
347 \text { colon cancer cases } \\
\text { and } 103 \text { rectal cancer cases } \\
(82 \%)\end{array}$ & $\begin{array}{l}\text { Ques- } \\
\text { tion- } \\
\text { naire at } \\
\text { baseline } \\
(1988)\end{array}$ & $\begin{array}{l}\text { Rotating night shifts; at least } \\
3 \text { nights/month, in addition to } \\
\text { days or evenings in that month } \\
(60 \%) \text {; total work history prior } \\
\text { to baseline }\end{array}$ & $\begin{array}{l}\text { Self-reported } \\
\text { cases confirmed } \\
\text { by medical re- } \\
\text { cords or national } \\
\text { death index }\end{array}$ & $\begin{array}{l}\text { Age, smoking, BMI, } \\
\text { physical activity, } \\
\text { aspirin use, screen- } \\
\text { ing endoscopy, diet, } \\
\text { alcohol, caloric intake, } \\
\text { hormone-replacement } \\
\text { therapy, height }\end{array}$ & $\begin{array}{l}\text { Duration } \\
\text { (years) } \\
\text { of rotat- } \\
\text { ing night } \\
\text { shifts: } \\
\text { never, } \\
1-14, \geq 15 \text {; } \\
\text { test for } \\
\text { trend of } \\
\text { continuous } \\
\text { term }\end{array}$ \\
\hline $\begin{array}{l}\text { Taylor } \\
\text { et al, } \\
1972(4) ; \\
\text { United } \\
\text { Kingdom }\end{array}$ & $\begin{array}{l}\text { All can- } \\
\text { cer }\end{array}$ & $\begin{array}{l}\text { Retrospective cohort of } \\
\text { industrial workers with } \\
>10 \text { years of employment, } \\
\text { 1956-1968; } 4188 ; 248 \text { cases } \\
(99.75 \%)\end{array}$ & Pay rolls & $\begin{array}{l}\text { Three-shift weekly rotating, 3- } \\
\text { shift rapid rotating, alternate day } \\
\text { and night }(99 \%)\end{array}$ & Death certificates & Age and calendar year & $\begin{array}{l}\text { No expo- } \\
\text { sure-re- } \\
\text { sponse } \\
\text { assess- } \\
\text { ment }\end{array}$ \\
\hline $\begin{array}{l}\text { Rafnsson } \\
\text { et al, } \\
1990(5) \text {; } \\
\text { Iceland }\end{array}$ & $\begin{array}{l}\text { All can- } \\
\text { cer }\end{array}$ & $\begin{array}{l}\text { Retrospective cohort of } \\
\text { workers at fertilizer plant, } \\
\text { 1954-1985; } 211 ; 10 \text { cases }\end{array}$ & Pay rolls & $\begin{array}{l}\text { Three shifts; total work history } \\
\text { in plant }\end{array}$ & $\begin{array}{l}\text { Mortality data } \\
\text { from national bu- } \\
\text { reau of statistics }\end{array}$ & Age and calendar year & $\begin{array}{l}\text { Duration } \\
\text { (years) }\end{array}$ \\
\hline
\end{tabular}

and Environmental Medicine (appendix 1). The key criterion was the epidemiologic evidence, while studies with experimental animals were considered to add only to the final classification.

\section{Results}

\section{Indivdual studies}

Breast cancer. Tynes et al (33) conducted a register- 
Table 3. Nightshift work and relative occurrence (R0) of cancer, overall results, and results by duration of nightshift work-findings of

\begin{tabular}{|c|c|c|c|c|c|c|c|c|c|c|c|}
\hline \multirow[t]{3}{*}{ Study } & \multicolumn{4}{|c|}{ Overall ${ }^{a}$} & \multicolumn{7}{|c|}{ Duration of nightshift work ${ }^{a}$} \\
\hline & \multirow[b]{2}{*}{ RO } & \multirow[b]{2}{*}{$95 \% \mathrm{Cl}$} & \multirow[b]{2}{*}{$\begin{array}{l}\text { Cases } \\
(\mathrm{N})\end{array}$} & \multirow[b]{2}{*}{ Exposure definition } & \multicolumn{3}{|c|}{ Reference } & \multicolumn{4}{|c|}{ Short duration } \\
\hline & & & & & Ro & $\begin{array}{l}\text { Cases } \\
(\mathrm{N})\end{array}$ & Duration definition & RO & $95 \% \mathrm{Cl}$ & $\begin{array}{l}\text { Cases } \\
(\mathrm{N})\end{array}$ & $\begin{array}{l}\text { Duration } \\
\text { definition }\end{array}$ \\
\hline \multicolumn{12}{|l|}{ Breast cancer } \\
\hline \multicolumn{12}{|c|}{ Tynes et al, 1996 (33); Norway } \\
\hline $\begin{array}{l}<50 \text { years of age } \\
\geq 50 \text { years of age }\end{array}$ & . & $\dot{r}$ & $\begin{array}{l}- \\
-\end{array}$ & $\dot{r}$ & $\begin{array}{l}1^{\mathrm{a}} \\
1^{\mathrm{a}}\end{array}$ & $\begin{array}{l}3 \\
1\end{array}$ & $\begin{array}{l}0 \text { years } \\
0 \text { years }\end{array}$ & $\begin{array}{l}0.9^{\mathrm{a}} \\
1.9^{\mathrm{a}}\end{array}$ & $\begin{array}{c}0.2-3.7 \\
0.2-17.9\end{array}$ & $\begin{array}{r}13 \\
5\end{array}$ & $\begin{array}{l}1-3.1 \text { years } \\
1-3.1 \text { years }\end{array}$ \\
\hline Davis et al, 2001 (32); US & $1.6^{\mathrm{a}}$ & $1.0-2.5$ & 54 & Ever nightshift & $1^{\mathrm{a}}$ & 733 & $<1$ nightshift/week & $1.4^{\mathrm{a}}$ & $0.6-3.2$ & 15 & $1-3$ years \\
\hline $\begin{array}{l}\text { Schernhammer et al, } \\
2001 \text { (22); US }\end{array}$ & . & . & - & . & $1^{b}$ & 925 & Never & $1.08^{b}$ & $0.99-1.18$ & 1324 & $1-14$ years \\
\hline $\begin{array}{l}\text { Hansen, } 2001 \text { (25); } \\
\text { Denmark }\end{array}$ & $1.5^{\mathrm{a}}$ & $1.3-1.7$ & 434 & $\geq 1 / 2$ year in selected occup & & 5847 & Daytime work & . & . & - & . \\
\hline $\begin{array}{l}\text { Lie et al, } 2005 \text { (34); } \\
\text { Norway }\end{array}$ & $\cdot$ & . & - & $\cdot$ & $1^{\mathrm{a}}$ & 50 & 0 years & $0.95^{\mathrm{a}}$ & $0.67-1.33$ & 362 & $1-14$ years \\
\hline $\begin{array}{l}\text { Schernhammer et al, } \\
2006 \text { (24); US }\end{array}$ & . & . & - & $\cdot$ & $1^{\mathrm{b}}$ & 441 & Never & $0.98^{b}$ & $0.87-1.10$ & 816 & $1-9$ years \\
\hline $\begin{array}{l}\text { O'Leary et al, } \\
2006 \text { (6); US }\end{array}$ & $0.55^{\mathrm{a}}$ & $0.32-0.94$ & 26 & Ever nightshift & $1^{\mathrm{a}}$ & 469 & $<1$ nightshift/week & $0.74^{\mathrm{a}}$ & $0.32-1.68$ & 11 & $1-8$ years \\
\hline $\begin{array}{l}\text { Schwartzbaum et al, } \\
2007 \text { (9); Sweden }\end{array}$ & $0.94^{c}$ & $0.74-1.18$ & 70 & $\begin{array}{l}\geq 20 \text { hours/week in } \\
\text { selected occup }\end{array}$ & $1^{c}$ & NR & $\begin{array}{l}<30 \% \text { shift workers } \\
\text { in occup }\end{array}$ & . & . & - & . \\
\hline \multicolumn{12}{|l|}{ Prostate cancer } \\
\hline \multicolumn{12}{|l|}{ Kubo et al, 2006 (7); Japan } \\
\hline Fixed night shifts & $2.3^{b}$ & $0.6-9.2$ & 3 & Longest form of work & . & - & . & . & . & - & . \\
\hline Rotating shifts & $3.0^{\mathrm{b}}$ & $1.2-7.7$ & 7 & Longest form of work & . & - & . & . & . & - & . \\
\hline $\begin{array}{l}\text { Conlon et al, } 2007 \text { (8); } \\
\text { Canada }\end{array}$ & $1.19^{a}$ & $1.00-1.42$ & 369 & Ever $\geq 1$ year nightshift & $1^{\mathrm{a}}$ & 391 & Never & $1.44^{\mathrm{a}}$ & $1.10-1.87$ & 115 & $1-6$ years \\
\hline $\begin{array}{l}\text { Schwartzbaum et al, } \\
2007 \text { (9); Sweden }\end{array}$ & $1.04^{c}$ & $0.99-1.10$ & 1319 & $\begin{array}{l}\geq 20 \text { hours/week in } \\
\text { selected occup }\end{array}$ & $1^{c}$ & c NR & $\begin{array}{l}<30 \% \text { shift workers } \\
\text { in occup }\end{array}$ & $\cdot$ & . & - & . \\
\hline \multicolumn{12}{|l|}{ Colon cancer } \\
\hline $\begin{array}{l}\text { Tynes et al, } 1996 \text { (33); } \\
\text { Norway }\end{array}$ & $1.3^{\mathrm{c}}$ & $0.6-2.6$ & 9 & $\begin{array}{l}\text { Ever radio or telegraph } \\
\text { operator }\end{array}$ & . & - & . & . & . & - & . \\
\hline $\begin{array}{l}\text { Schernhammer et al, } \\
2003 \text { (23); US }\end{array}$ & . & . & - & . & $1^{b}$ & 137 & Never & $0.93^{b}$ & $0.74-1.17$ & 169 & $1-14$ years \\
\hline $\begin{array}{l}\text { Schwartzbaum et al, } \\
2007 \text { (9) }\end{array}$ & $1.03^{c}$ & $0.94-1.13$ & 449 & $\begin{array}{l}\geq 20 \text { hours/week in } \\
\text { selected occup }\end{array}$ & $1^{c}$ & - & $\begin{array}{l}<30 \% \text { shift workers } \\
\text { in occup }\end{array}$ & . & . & - & . \\
\hline \multicolumn{12}{|l|}{ All cancer } \\
\hline Taylor et al, 1972 (4); UK & $1.16^{\mathrm{d}, \mathrm{e}}$ & . & 219 & $\geq 10$ years of shift work & . & - & . & . & . & - & . \\
\hline $\begin{array}{l}\text { Rafnsson et al, } 1990 \text { (5); } \\
\text { Iceland }\end{array}$ & $1.40^{\dagger}$ & . & 14 & $\geq 1$ year shift work & . & - & . & $4.12^{f}$ & . & 4 & $\leq 1$ year \\
\hline $\begin{array}{l}\text { Tynes et al, } 1996 \text { (33); } \\
\text { Norway }\end{array}$ & $1.2^{\mathrm{c}}$ & $1.0-1.4$ & - & $\begin{array}{l}\text { Ever radio or telegraph } \\
\text { operator }\end{array}$ & . & - & . & . & . & - & . \\
\hline $\begin{array}{l}\text { Schwartzbaum et al, } \\
2007 \text { (9); Sweden }\end{array}$ & $1.02^{\mathrm{c}}$ & $1.00-1.05$ & 6524 & $\begin{array}{l}\geq 20 \text { hours/week in } \\
\text { selected occup }\end{array}$ & $1^{c}$ & c NR & $\begin{array}{l}<30 \% \text { shift workers } \\
\text { in occup }\end{array}$ & . & . & - & . \\
\hline
\end{tabular}

linked case-control study among 2619 women recorded in a national register of certified radio and telegraph operators who had primarily worked in the merchant navy. A total of 50 incident breast cancers [International Classification of Diseases 7 th revision (ICD-7) 170] were identified in a national cancer register, and
259 age-matched controls were drawn from within the study population. Histories of employment on ships were obtained from a seamen register, and a shipping journalist and a researcher with detailed knowledge of the merchant navy classified each ship with respect to shift work (4 categories: 0, 1, 2, 3). It was stated that 
13 epidemiologic studies, 1972-2007. [US = United States, occup = occupation(s), UK = United Kingdom, NR = not reported]

\begin{tabular}{|c|c|c|c|c|c|c|c|c|c|c|c|c|}
\hline \multicolumn{12}{|c|}{ Duration of nightshift work (continues) a } & \multirow{3}{*}{$\begin{array}{c}\text { Test for } \\
\text { t-trend } \\
\text { (P-value) }\end{array}$} \\
\hline \multicolumn{4}{|c|}{ Medium duration } & \multicolumn{4}{|c|}{ Long duration I } & \multicolumn{4}{|c|}{ Long duration II } & \\
\hline Ro & $95 \% \mathrm{Cl}$ & $\begin{array}{l}\text { Cases } \\
(\mathrm{N})\end{array}$ & $\begin{array}{l}\text { Duration } \\
\text { definition }\end{array}$ & RO & $95 \% \mathrm{Cl}$ & $\begin{array}{l}\text { Cases } \\
(\mathrm{N})\end{array}$ & $\begin{array}{l}\text { Duration } \\
\text { definition }\end{array}$ & Ro & $95 \% \mathrm{Cl}$ & $\begin{array}{c}\text { Cases } \\
(\mathrm{N})\end{array}$ & $\begin{array}{l}\text { Duration } \\
\text { definition }\end{array}$ & \\
\hline
\end{tabular}

\begin{tabular}{|c|c|c|c|c|c|c|c|c|c|c|c|c|}
\hline $0.8^{\mathrm{a}}$ & $0.2-3.6$ & 13 & $3.2-14.6$ years & . & . & - & . & . & . & .. & . & 0.8 \\
\hline $5.9^{\mathrm{a}}$ & $0.7-47.7$ & 15 & $3.2-14.6$ years & . & . & - & . & . & . & .. & . & 0.0 \\
\hline $1.6^{\mathrm{a}}$ & $0.8-3.2$ & 19 & $\geq 3$ years & . & . & - & . & . & . & .. & . & \\
\hline $1.08^{b}$ & $0.90-1.30$ & 134 & $15-29$ years & $1.36^{\mathrm{b}}$ & $1.04-1.78$ & 58 & $\geq 30$ years & . & . & .. & . & \\
\hline $1.7^{\mathrm{a}}$ & $1.3-1.7$ & 63 & $>6$ years & . & . & - & . & . & . & .. & . & \\
\hline $1.29^{a}$ & $0.82-2.02$ & 101 & $15-29$ years & $2.21^{a}$ & $1.10-4.45$ & 24 & $\geq 30$ years & . & . & .. & . & \\
\hline $0.91^{\mathrm{b}}$ & $0.72-1.16$ & 80 & $10-19$ years & $1.79^{b}$ & $1.06-3.01$ & 15 & $\geq 20$ years & . & . & .. & . & \\
\hline $0.32^{\mathrm{a}}$ & $0.12-0.83$ & 6 & $\geq 8$ years & . & . & - & . & . & . & .. & . & \\
\hline $0.97 \mathrm{c}$ & $0.67-1.40$ & 28 & $>10$ years & . & . & - & . & . & . & .. & . & \\
\hline
\end{tabular}

$\begin{array}{llllllllllllll}1.14^{\text {a }} & 0.86-1.52 & 87 & 7-21 \text { years } & 0.93^{\text {a }} & 0.70-1.23 & 81 & 22-33 & 1.30^{\text {a }} & 0.97-1.74 & 86 & \geq 34 \text { years } & 0.42 \\ 1.02^{\mathrm{c}} & 0.95-1.10 & 780 & >10 \text { years } & . & . & - & . & . & . & & . . & . & \\ \end{array}$

$\begin{array}{llll}1.32^{\mathrm{b}} & 0.93-1.87 & 41 & \geq 15 \text { years } \\ & & & \\ 1.02^{\mathrm{c}} & 0.90-1.15 & 266 & >10 \text { years }\end{array}$

\begin{tabular}{|c|c|c|c|c|c|c|c|c|c|c|c|}
\hline $2.02^{\dagger}$ & . & 2 & $2-5$ years & $1.71^{\dagger}$ & . & 5 & $6-15$ years & $0.59^{\dagger}$ & . & 3 & $\geq 16$ years \\
\hline . & . & - & . & . & . & - & . & . & . & .. & . \\
\hline $1.01^{c}$ & $0.98-1.05$ & 3799 & $>10$ years & . & . & - & . & . & . & .. & . \\
\hline
\end{tabular}

e $\mathrm{P}<0.05 . \quad$ f Standardized mortality ratio.

this classification reflected frequent presence in the radio room both at night and during the day. The shiftwork variable was multiplied by the number of years employed within each category (this information was however not clearly stated), and this index was used together with the duration of employment in the analyses. The analyses were adjusted for a fertility variable (no children, age $<25$ years at birth of first child, age $\geq 25$ years at birth of first child), but only for participants born in 1935 or later (this information was available for 6 out of 21 cases $>50$ years of age; apparently information was complete for participants $<50$ years of age). Data were 
presented separately for women $<50$ years of age and those $\geq 50$ years of age.

The odds ratio $(\mathrm{OR})$ of breast cancer increased by duration of employment (P-value $=0.02)$, and, for the operators employed for more than 3.1 years (the highest category), an odds ratio of 5.9 [95\% confidence interval (95\% CI) 0.7-47.7] was observed when the group was compared with operators never undertaking nightshift work among those aged $\geq 50$ years. Similar findings were found if the odds ratio was analyzed according to the cumulative index.

For the operators who were not yet 50 years of age, there was no excess. Comparable results were found in analyses including only work histories before the age of 30 years.

Davis et al (32) conducted a population-based casecontrol study of nightshift work and breast cancer. The case patients were women aged 20-74 years, diagnosed with breast cancer [International Classification of Diseases for Oncology (ICD-O) 174.0-174.9] between 1992 and 1995, and identified by the SEER (surveillance, epidemiology, and ends results) cancer surveillance program. Age- and sex-matched controls were identified by random-digit dialing. Altogether $78 \%$ of the cases and $75 \%$ of the controls agreed to participate. Through individual interviews a lifetime occupational history was obtained that included any job lasting 6 months or longer. For each job, the percentage of time worked during the day, during the evening, and on the graveyard shift was requested. The graveyard shift was defined as "beginning work after 1900 and leaving work before 0900 ". For the 10 years prior to a diagnosis, the following three variables further characterized the graveyard shift: (i) ever worked the graveyard shift, (ii) hours per week on the graveyard shift, and (iii) number of years of at least one graveyard shift per week.

The adjusted odds ratio for breast cancer for ever working the graveyard shift during the 10 years prior to a diagnosis was 1.6 (95\% CI 1.0-2.5). The risk increased by number of hours per week on the graveyard shift (P-value, test for trend 0.03 ) and by the number of years with at least one graveyard shift per week (Pvalue, test for trend 0.04 ) within this 10 -year period. These analyses were adjusted for parity, family history of breast cancer, oral contraceptive use, and hormone replacement therapy.

No results were presented for lifetime work history.

Schernhammer et al (22) studied the risk of breast cancer following work on rotating nightshifts among nurses participating in the United States (US) Nurses' Health Study. The study enrolled 121701 female nurses aged 30-55 years in 1988. After exclusions were made for those with incomplete data or previous cancer, 78562 nurses comprised the study population that was followed until 1998. The participants in 1988 completed a questionnaire that included questions about nightshift work that was defined as "rotating night shifts with at least three nights per month in addition to days or evenings". The participants reported whether or not they had been diagnosed with breast cancer, and the national death index was searched for additional cases. Altogether a total of 2441 cases of breast cancer were ascertained between 1988 and 1998. Pathology records were identified for $93 \%$.

The risk of breast cancer, adjusted for a comprehensive list of known, potential confounders, increased as the number of years on rotating shifts increased up to 1988 in a comparison with those who never had such work prior to 1988 (P-value for trend 0.02). For those with more than 30 years on rotating shifts, the adjusted relative risk (aRR) was 1.36 (95\% CI 1.04-1.78). Comparable results were found for pre- and postmenopausal women. Risk estimates adjusted for age only were almost identical to those obtained in the full models.

Hansen (25) reported breast cancer risk among women employed in the manufacture of beverages, in land transport services, in catering, and in air transport services in a register-linked nested case-control study. Women aged 30-54 years $(\mathrm{N}=7035)$ with breast cancer were identified in the national cancer register. Individually matched controls were obtained from a national population register. Employment histories since 1964 were obtained for the cases and controls from a national pension fund. A survey conducted in 1976 showed the highest prevalence of female workers with nighttime schedules (64-71\%) in these four trades.

The odds ratio for breast cancer among women ever employed for $>6$ months in these trades was 1.5 (95\% CI 1.3-1.7) when they were compared with women employed in trades with less than $40 \%$ female employees working nighttime schedules according to the survey. Trades with 40-60\% female nighttime workers were excluded from the analyses. The analyses were adjusted for the number of children, age at birth of first child and age at birth of last child, and current job title. The 1976 survey showed that the average number of alcoholic drinks consumed was substantially higher (3-6 times the median value for all female employees) in the four exposed trades. It was stated that there was a positive trend towards breast cancer risk according to the duration of work at night, but only the results for those employed for $>6$ years in the exposed trades were presented (OR 1.7, 95\% CI 1.3-1.7). Taking account of time since first employment did not influence the risk estimates substantially.

Lie et al (34) studied 537 breast cancer cases and 2143 individually matched controls in a register-linked study within a cohort of 44853 female nurses registered in a national register of nurses. The register included nurses who had graduated from a nursing school in 
Norway between 1914 and 1980 and were alive in 1949 or born later. The cases were identified in the national cancer register (ICD-7 codes). For each case, four controls alive and without breast cancer at the time the cases were diagnosed were randomly drawn from the cohort and individually matched by year of birth.

Work histories were reconstructed from the register of nurses. This register included self-reported information on workplace and, to some extent, ward or department for the period prior to 1968 (the last regular update) and only sporadically thereafter. Additional data were obtained from 1960, 1970, and 1980 censuses if the occupational code was "nursing" or "nursing and other care work" or the industry code was "health work". All work at infirmaries was defined as night work, except for managerial jobs, teaching, and work in physiotherapy or outpatient departments. Information on workplace was incomplete after 1960 because the censuses did not include information on workplace.

The odds ratios were analyzed using a conditional logistic regression adjusted for age at birth of first child, number of children, and total employment time. The adjusted odds ratio for breast cancer increased as the years with night work increased, as defined by the authors, and the risk was increased twofold if night work had lasted $\geq 30$ years as compared with no night work. A test for trend showed a P-value of 0.01. A similar pattern was found if the last 20 years of employment were disregarded.

Schernhammer et al (24) analyzed rotating night shifts and the relative risk of breast cancer in the Nurses' Health Study II that enrolled 115022 nurses aged 25-42 years in 1989. The population did not overlap that studied by Schernhammer in 2001 (22). The participants filled out questionnaires about night work in 1989, 1991, 1993, and 1997 and retrospectively in 2001 for the time periods 1993-1995 and 1997-1999. Rotating nightshift work was defined as in the previous report (22). Questions were asked about months working rotating nightshifts in six categories $(0,1-4,5-9,10-14,15-19$ and $\geq 20$ months) and permanent night shifts for $\geq 6$ months. In the analyses the participants were classified by the lifetime total number of years they had worked rotating night shifts until the date of diagnosis. Other procedures were comparable with the analyses based on the Nurses' Health Study I. Nurses who reported $>20$ years of rotating nightshift work showed an elevated relative risk (RR) of breast cancer when compared with nurses never working rotating nightshifts (RR 1.79, 95\% CI 1.06-3.01) after adjustment for multiple potential confounding factors (but this procedure did not change the results substantially from the age-adjusted RR estimates). There was no increased risk with fewer years of working on rotating nightshifts. No results were presented for permanent nightshift work.
O'Leary et al (6) conducted a population-based case-control study of breast cancer in 1996-1997 in Long Island, New York. They included 467 cases and 509 controls recruited through a multistep procedure, and the participation rates were not easily computable. The cases were newly diagnosed patients with first primary invasive or in situ breast cancer identified by weekly contacts to hospitals, pathology departments, and physicians in the study area (80). Occupational histories were obtained for all of the jobs held for $\geq 6$ months during the 15 years prior to the diagnosis (cases) or reference (controls) date. The frequency (days per week, month, and year), duration, and type of shift work were ascertained for each job, and the participants were specifically asked about overnight shifts defined as "starting as early as 1900 and continuing until the next morning". Among the cases 5.3\%, and among the controls $9.8 \%$, had ever worked overnight shifts during the previous 15 years.

The odds ratio for breast cancer, adjusted for age, parity, family history, education, and previous benign breast disease, was 0.55 (95\% CI 0.32-0.94) for any overnight shift work and 0.64 (95\% CI $0.28-1.45$ ) for overnight shift work but no evening shift work. The corresponding crude odds ratio estimates were 0.53 (95\% CI $0.32-0.88$ ) and 0.57 (95\% CI $0.26-1.25)$, respectively. The adjusted odds ratios decreased by duration of overnight shift work (only periods with $>1$ night shift/week were included) and an odds ratio of 0.32 (95\% CI $0.12-0.83)$ was found for the longest exposure category ( $\geq 8$ years). The odds ratio for breast cancer following evening shift work was 1.21 (95\% CI 0.90-1.64), but, according to the melatonin hypothesis, evening work should not be a risk factor for breast cancer (1).

Schwartzbaum et al (9) analyzed all major cancer sites in a cohort study of all gainfully employed inhabitants of Sweden ( $\geq 20$ hours/week) included in the 1960 and the 1970 censuses. Altogether 3\% of the men and $0.3 \%$ of the women were classified as shift workers (69 759 men and 3057 women). Among them, 6792 incident cases of cancer were identified in the national cancer register during the follow-up period, 1970-1989. The classification of shift workers relied on a job-exposure matrix constructed from a survey of living conditions in 1977-1981. Shift workers were defined by occupation-industry combinations recorded in the censuses with at least $40 \%$ working rotating shifts with three or more possible shifts per week or working any hour between 1300 and 0400 at least 1 day per week. The most common exposed occupations were paper and paperboard worker, paper pulp worker, furnace worker, fire fighter, policeman, civilian protective service worker, and railway engine driver among the men and crane and hoist operator, delivery woman in the paper and printing industry and midwife among the women. The reference 
population was occupation-industry combinations with $<30 \%$ shift workers.

The observed number of breast cancer cases was close to the expected [standardized incidence ratio (SIR) $0.94,95 \%$ CI $0.74-1.18]$. This was also the case when the analyses were restricted to workers employed in a job classified as shift worker in both 1960 and 1970 and when only workers of jobs with $>70 \%$ shift workers were classified as exposed.

Prostate cancer. Kubo et al (7) studied 14052 men working between 1988 and 1990 and followed them until 1997. In a self-administered questionnaire, the participants were asked which work schedule they had been engaged in the longest. Altogether 20\% reported fixed or alternate night and day work. Incident cases of prostate cancer (ICD-10 C61, N=31) were identified by linkage with several regional cancer registers. The analyses were adjusted for a long list of potential confounders, and the reference was daytime work. The study showed relative risks of 2.3 (95\% CI 0.6-9.2) for fixed night work and 3.0 (95\% CI 1.2-7.7) for alternate night and day work. No analyses were conducted for the duration of nightshift work.

Conlon et al (8) utilized previously collected population-based case-control data to assess a possible association between shift work and prostate cancer. A total of 760 cases recorded with a cancer register diagnosis of prostate cancer and 1632 controls reported their lifetime work history and, for each job lasting $\geq 1$ years, described the usual worktime as "daytime shift, evening or night shift, rotating shift, other". The participants were classified as ever having worked full-time rotating shifts (excluding those working part-time rotating shifts) and according to the duration of this work. Among the controls, $44 \%$ reported ever working fulltime-rotating shifts. The analyses were adjusted for age and family history of prostate cancer. The odds ratio for prostate cancer was 1.19 (95\% CI 1.00-1.42) for ever working rotating shifts. There was no trend by years of rotating shifts $(\mathrm{P}$-value $=0.42)$.

Schwartzbaum et al (9) reported no increased risk of prostate cancer (SIR 1.04, 95\% CI 0.99-1.10). Further details of this study were presented earlier in this review.

Colon cancer. Tynes et al (33), in addition to detailed results for breast cancer, reported standardized incidence ratios for the most frequent cancer sites among all radio and telegraph operators using the background population as reference and showed a nonsignificantly increased risk for colon cancer (SIR 1.3, 95\% CI 0.6-2.6). Further details of this study were presented earlier in this review.

Schernhammer et al (23) studied the risk of colon and rectum cancer following rotating nightshifts in the US Nurses' Health Study, and the study population was almost identical to the population of Schernhammer et al's in 2001 (22). The participants with ulcerative colitis, Chron's disease, or familial polyposis syndrome, in addition to a previous cancer (except nonmelanoma skin cancer), were excluded, and the population then comprised 78586 participants. Information on nightshift work was identical to the information of the 2001 study. The analyses were adjusted for an extensive list of potential confounders and showed that women who worked 1-14 or $\geq 15$ years on rotating night shifts had relative risks of 0.93 (95\% CI 0.74-1.17) and 1.32 (95\% CI 0.93-1.87), respectively, for colon cancer. A test for trend showed a P-value of 0.20 . When rectal cancer was included in the case category, a similar pattern was found, but the trend test reached statistical significance $(\mathrm{P}$-value $=0.04)$.

Schwartzbaum et al (9) reported no increased risk of colon cancer (SIR 1.03, 95\% CI 0.94-1.13).

All cancer. Taylor et al (4) included 4188 male workers employed for at least 10 years in shift work in, for example, coal, brick, metal, and vehicle manufacturing in a retrospective cohort study in 1956 to 1986. Altogether $45 \%$ worked three-shift weekly rotating shifts, $35 \%$ worked three-shift rapid rotating shifts, and 19\% worked alternate days and nights. A total of 722 died during the follow-up, and 219 died from cancer, while 188.8 deaths were expected according to the age- and calendar-year-adjusted national rates, P-value $<0.05$. This value corresponds with a standardized mortality ratio of 1.16. Results were also presented for ex-shift workers who did not fulfill the 10-year night shiftwork criterion; the standardized incidence ratio was then 1.12 ( 29 observed cancer deaths).

Rafnsson et al (5) conducted a retrospective cohort study of 211 men employed in a fertilizer plant for at least 1 year in 1954-1985 and followed them until 1985. They all worked three shifts according to payroll information, and 34 died during the follow-up, 14 due to cancer, while 9.98 deaths were expected from the national rates. The mortality decreased as the years of shift work increased.

Tynes et al (33) reported a standardized incidence ratio of 1.2 (95\% CI 1.0-1.4) for all cancer among all radio and telegraph operators using the background population as reference.

Schwartzbaum et al reported a marginally increased overall cancer risk of statistical significance among workers classified as nightshift workers (SIR 1.02, 95\% CI 1.00-1.05) (9).

\section{Contributory evidence}

Light at night at home and latitude of residence. Some epidemiologic studies have suggested that light at night at home is associated with breast cancer. Davis and 
his colleagues (32) showed an increased risk among women who did not sleep when the nocturnal melatonin peak typically occurs, and O'Leary et al (6) found an increased risk among women who frequently turned on the light at home during sleep hours. However, both studies relied on self-reported retrospective information about light exposure, and information bias was a likely explanation for the findings. Lower breast cancer incidence has been reported in the arctic region, and it has been hypothesized that lower light exposure during wintertime may at least partly be a causal link (65).

Pilots and flight attendants. Flight attendants have an increased risk of breast cancer, and it has been suggested that this increment is related to exposure to light at night (3). However, the studies published so far have not assessed nightshift work specifically. Pukkala et al (81) reported an increased incidence of prostate cancer among male airline pilots that increased as the number of long-haul flights (expected to include nightshift work and jet lag) increased, but the risk also increased as the dose of cosmic radiation increased, whereas the allcancer and colon-cancer incidence did not exceed the expected numbers. In a large European study of airline cabin attendants, mortality from all cancers and largeintestine cancer was lower for both genders than for the background population (82). Mortality from breast cancer was slightly increased among the women but showed no trend by duration of employment. Rafnsson et al (83) reported an increasing risk of breast cancer as the length of employment increased among Icelandic cabin attendants flying long-haul international flights in the pre-jet period, but no trend was evident after jet planes were introduced. Kojo et al (68) showed no evidence that the risk of breast cancer increased as the number of long-haul flights increased among Finnish female cabin attendants.

Cancer risk and melatonin in the visually impaired. The risk of breast cancer and possibly also prostate cancer seems to decrease as the degree of visual impairment increases (84). Blind persons may show abnormal melatonin rhythms (85), but the level of melatonin output during a 24-hour period, measured by 6-sulphatoxymelatonin, does not correlate with the level of visual impairment $(86,87)$.

Melatonin level and risk of breast cancer. In a prospective study, urinary concentrations of 6-sulfatoxymelatonin in 24-hour urine samples did not differ between breast cancer patients and their controls (88), while, in another prospective study, the odds ratio for breast cancer declined as the levels of 6-sulfatoxymelatonin increased in morning urine samples (76). These two studies are the only prospective studies published on this issue so far.
Biological mechanisms. Originally, it was hypothesized that light at night could suppress melatonin output and increase estrogen levels and thereby increase the risk of breast cancer, but, at present, it is unclear if light at night or melatonin affects estrogen levels in humans (2). Melatonin has been shown to have an oncostatic effect on chemically induced tumors with an increased tumoral latency (the time elapsing from the administration of the carcinogen and the appearance of palpable mammary tumors) among rodents, and constant light has stimulated tumorigenesis in rats, but not in all studies $(36,55)$. In a frequently cited study by Blask et al (89), constant light increased the growth of a human breast cancer xenograft implanted into a nude rat model. Melatonin has also been proposed to act as an antiestrogen, to enhance immune function, and to have antioxidant properties (90). Anderson et al (91) reported a significant reduction in the number of breast tumors among female rats that were exposed to constant light from the age of 26 days on, and this result was in contrast to earlier findings from rats exposed to constant light beginning before birth. In addition, Stevens (2) has hypothesized that light exposure in utero may increase breast cancer risk.

\section{Discussion}

\section{Overall findings}

Years of nightshift work was the principal measure of exposure analyzed across the studies in this review, except for the studies authored by Kubo et al (7) and Taylor et al (4). Three studies reported a significantly increased risk of breast cancer for long-term nightshift work beyond $20-30$ years $(22,24,34)$. Virtually no effects were found for shorter durations. The two studies by Hansen (25) and Davis et al (32) showed a small influence of duration of employment on the risk of breast cancer, but these authors defined long-term shift work as 3 or $\geq 6$ years, respectively. However, both studies reported an increased risk of breast cancer in the overall analyses (ever versus never) of statistical significance. O'Leary et al (6) showed a negative trend for increasing years of nightshift work (no statistical testing of the trend was presented). Others reported trend tests of statistical significance for duration of nightshift work $(22,32,34)$. However, these tests have to be interpreted with caution since increased risks in single categories may have influenced the P-values heavily. Furthermore, it was not always clear if the tests were based on the original continuous exposure data or on the categorical classification (eg, the midpoint values).

Tynes et al (33) observed an increasing risk for breast cancer for those above 50 years of age but not for those below this age; this finding was not replicated by Lie et al (34). Shernhammer et al (22) reported comparable 
risks for premenopausal and postmenopausal nurses, and menopausal status probably does not modify the risk of breast cancer following nightshift work.

Kubo et al (7) and Conlon et al (8) indicated increased risks for prostate cancer for both fixed and rotating night shifts, but the cases were few or the effect was only marginal. Schernhammer et al (23) reported an increasing risk of colon cancer as the duration of rotating night shifts increased, and this finding was supported by the slightly increased standardized incidence ratio observed by Tynes et al (33) for all radio and telegraph operators. However Schwartzbaum et al (9) observed no increased risk of colon cancer. The occurrence of all cancer was close to the expected values in the four studies that analyzed this outcome (RR 1.0-1.2) (4, 5, 9, 33). A negative exposure-response relationship was indicated in the only study that assessed the duration of shift work, and this finding does not support a causal effect due to occupational factors (5).

\section{Study populations and design}

Of the 13 studies, 6 were based on the general population, 4 studied nurses (three independent populations), 2 studied industrial workers, and 1 studied radio and telegraph operators.

It was noted that the 3 studies indicating a long-term effect of nightshift work on breast cancer risk were all conducted among nurses $(22,24,34)$. Four studies (three populations) used a prospective cohort design ( 7 , 22-24), four were register-linked studies $(9,25,33,34)$, three were case-control studies $(6,8,32)$, and two were retrospective cohort studies linking personnel records and mortality data $(4,5)$.

\section{Cancer outcome}

Cancer diagnoses relied on cancer register data, weekly reports from diagnosing institutions, medical records, and death certificates and were expected to have high specificity in all of the studies and to be unrelated to exposure status. The identification of cases in the three studies of Schernhammer et al (22-24) did, however, rely partly on self-reports and may have been affected by nightshift status, but this possibility is less likely.

\section{Nightshift work}

O'Leary et al (6) defined nightshift work the most precisely. The definition of Davis et al (32) was less specific and did not request that the shift last overnight. Schernhammer et al (22), Kubo et al (7), and Conlon et al (8) did not request that the participants consider the timing or duration of their nightshift work during the day.

O'Leary et al (6) and Davis et al (32) classified the participants as exposed in the principal analyses if they had experienced one nightshift per week or more, which corresponds well with the 3 days per month criterion applied by Schernhammer et al (22).

Schernhammer et al (22) did not include nurses working fixed (permanent) night shifts in their exposed category. If fixed nightshift workers have an increased risk of breast cancer and furthermore were classified as unexposed, the results would be biased towards the null. If, on the other hand, fixed nightshift workers have no increased risk, there would be inconsistent findings if the cumulative number of nightshifts is the relevant exposure metric. No increased risk of breast cancer for the fixed nightshift workers could also have been the finding because these workers adopt an altered circadian rhythm and melatonin is less strongly suppressed. But this is not a likely possibility since fixed nightshift workers are expected to return to a normal diurnal rhythm during weekends and days off. Under all circumstances, we would have appreciated having results for both rotating and fixed nightshift workers.

Given the low prevalence of workers on fixed nightshifts among all nightshift workers [0.4\% versus $20 \%$ (18)], it was expected that the reviewed studies mainly analyzed the effect of nonfixed (or rotating) nightshift work, even if they did not specify such. One exception is the study by Kubo et al (7) that reported results for fixed nightshift work. The present database thus does not allow any assessment of a possibly different risk profile for fixed night shifts.

In the population-based studies, the prevalence of nightshift work was $3 \%$ for the men and $0.3 \%$ for the women in the study by Schwartzbaum (9), $5 \%$ for the women in the studies of Davis et al (32) and Hansen (25), and $20 \%$ and $44 \%$, respectively, in the studies by Kubo et al (7) and Conlon et al (8). These differences probably reflect different work conditions across the study populations. They also reflect the different definitions of nightshift work used, and the very low prevalence in the study by Scwartzbaum et al (9) may cause the validity of their classification scheme to be doubted.

As expected, the cut-off levels used to define increasing levels of nightshift work differed across studies, but it was noted that this approach was also the case for the three studies authored by Schernhammer et al (22-24). It would be of interest to see all of the results lined up according to the same cut-off points.

Information about nightshift work was obtained retrospectively in the three case-control studies $(6,8,32)$, and thus the reporting may have been influenced by case status. However, shift work is easily estimable, and information bias probably has not been a major concern. On the other hand, selective recall may have significantly affected the results for ambient light levels at night in homes.

In the four register-linked studies, nightshift work was assessed on a group level (ship, industry, type of 
hospital department, and occupation-industry combinations) and thus represented estimates of the likelihood of nightshift work (eg, an estimated $40 \%$ of the participants classified as exposed in the study by Hansen were not involved in nightshift work) (25). Lie et al (34) and Tynes et al (33) did not assess the degree of misclassification of nightshift work, and Tynes et al did not differentiate between evening shifts and night shifts. Schwartzbaum et al (9) apparently included an unknown proportion of workers on evening shifts in the exposed category of their principal analyses. More than $60 \%$ of the workers of this study classified as exposed were furthermore not engaged in shift work. These studies compared ships, hospital departments, occupations, and industries, which are proxy measures of nightshift work that may have low specificity. Such misclassification of exposure is expected to be nondifferential with respect to cancer status and thus will bias true associations towards the null. On the other hand, it is likely that such rather broad proxy measures may be proxies for other exposures and not only nightshift work. The Hansen study (25) exemplifies this possibility by showing that the manufacture of beverages, land transport services, catering, and air transport services (proxies for nightshift work) are also proxies for alcohol consumption. The results of these studies thus may have been confounded in any direction and not necessarily towards the null. On the other hand, these studies are robust with respect to information bias and selection bias due to the complete register data from independent sources.

Nightshift work per se is not expected to be a risk factor for cancer; but is, instead, a surrogate measure for, for example, light at night or circadian phase shift or is an influence on an intermediary risk factor (eg, sleep deprivation, diet or lifestyle that is causally related to cancer). In any case, one would expect that cancer risk increases as the cumulative number of nightshifts increases, regardless of whether this work entails fixed nightshifts or rotating nightshifts. Workers with fixed nightshifts are expected to accumulate a higher number of shifts than rotating nightshift workers, and excluding them from the analyses would thus reduce the power to detect a true effect. In line with this reasoning, Kubo et al (7) showed slightly higher risks of prostate cancer for fixed nightshift work than for rotating nightshift work. It would therefore be of interest to have data presented for the fixed nightshift workers of the Nurses Health Study II (24) and also data based on the lifetime working histories of Davis et al's study (32).

It has been argued that workers on rotating or mixed night shifts should have the highest cancer risk because they do not retrain to an altered circadian rhythm (22). However, a large field study of the melatonin profile of nightshift workers showed lower melatonin concentrations in fixed nightshift workers than in mixed nightshift workers (29). Offshore workers working 2 weeks on 12-hour night shifts adapted to an altered circadian rhythm and showed rates of phase shifts of 1-2 hours per day (92). However, we have not been able to identify field studies of melatonin profiles among nurses or others working a limited number of nights per week that documented that mixed or rotating nightshift workers have more irregular circadian phases than day workers or permanent nightshift workers.

\section{Confounding}

There is evidence indicating that the nightshift workers included in this review more often smoked, had a higher body mass index, and less often had hormone replacement therapy than dayshift workers. Smoking is not an independent risk factor for breast cancer but may be a proxy for other factors related to lifestyle and socioeconomic status that may be risk factors for breast cancer (16). The inverse relationship between socioeconomic status and breast cancer risk does not, however, support this argument (93). A higher prevalence of women with early menarche was indicated among nightshift workers, but they generally showed no consistent differences when compared with day workers observed with respect to reproductive factors; however, the differences were found within individual studies that should be accounted for in the adjusted analyses.

The workers classified as nightshift workers in the Hansen study consumed up to six times more alcohol than the day workers (3.5-6.0 drinks/week versus 1.1 drinks/week), but alcohol can only explain a few percent of the observed increased risk of breast cancer (16) and has barely confounded the results significantly.

The three studies that suggested an effect of longterm nightshift work were all conducted among nurses $(22,24,34)$. On one hand, this similarity may have been a strength because extraneous risk factors are expected to be less of a problem in analyses relying on comparisons within a profession. On the other hand, this likeness may also have been a major limitation since these nightshift nurses may have shared common, yet unknown, risk factors other than nightshift work, and I would have felt much more confident if the results had been replicated across different occupations and industries.

Confounder control was limited in the four register-based studies because routine register data on, for example, body mass index, oral contraceptive use, hormone replacement therapy, and age at menarche and menopause were not available $(9,25,33,34)$. Several studies did not control for oral contraceptive use or hormon replacement therapy, and Schernhammer et al were the only ones to adjust for alcohol consumption (22-24). Still it has to be emphasized that no strong confounding effect was indicated by the studies of Schernhammer et 
al $(22,24)$ or O'Leary et al $(6)$, who presented crude risk estimates in addition to the adjusted estimates.

One also has to bear in mind that, if alcohol or smoking habits or other cancer risk factors are influenced by nightshift work (act as intermediary risk factors), adjusting by these factors may blur causal relationships.

\section{Selection bias}

The participation rates were generally high in all of the studies, and selective study participation is not expected to have influenced the findings significantly, especially in the case of the register-linked studies with almost complete data. The Davis et al study (32) may be an exception, since these authors identified controls by random-digit dialing that may possess specific problems when nightshift work is studied, since night working controls may not be reached by telephone as often as day workers.

On the other hand, selective reporting of findings within the individual studies may have influenced the overall risk pattern obtained for nightshift work. For instance, Schernhammer et al (23) presented results for colon and rectal cancer but not for other cancer sites even if they argued in favor of a general effect on the tumorigenesis of light at night, and this information was collected in the Nurses Health Study (94). Furthermore, Schernhammer et al (24) and Conlon et al (8) obtained data on fixed shift work, and Davis et al (32) collected data on lifetime nightshift work, but none of them presented the findings for these exposure measures. Hansen (25) left workers of industries with an estimated 40-60\% nightshift workers out of the analyses. Schwartzbaum et al (9) did the same to those with 30-40\% shift workers. If the decisions as to whether results should be presented or not were made a priori, the results should not have been affected. But if the decisions were data driven, the collective risk pattern might have been biased. I am, of course, aware that numerous decisions have to be made when data are extracted, analyzed, and presented, and much information must be left out due to parsimonious results and clear conclusions. However, I would have appreciated it if these decisions had been more thoroughly described and the nonpresented results briefly mentioned.

\section{Contributory evidence}

The supportive database in favor of a causal association between light at night and breast cancer is large, but still there are numerous conflicts. Animal experiments have documented significant oncostatic effects of melatonin, and constant light exposure stimulates mammary carcinogenesis. But it has been emphasized that results based on nocturnal animals should only be generalized to diurnal humans with caution (40). Furthermore, one may question whether the exposure models are valid for nightshift work. Thus it is uncertain to what degree the results can be extrapolated to humans working night shifts.

Nightshift workers have lowered melatonin levels during night shifts and may adapt to an altered circadian rhythm during longer periods of nightshift work, but the total 24-hour melatonin output is apparently not significantly affected by nightshift work, at least among nurses (the profession most extensively studied for breast cancer risk) working mixed and fixed night shifts. Airline crew are expected to experience jet lag and nightshift work during long-haul flights and have an increased risk of breast cancer, and possibly also of prostate cancer. But data based on individual flight histories are sparse and equivocal.

The three positive studies of breast cancer were restricted to nurses $(22,24,34)$. A high proportion of nurses work rotating night shifts $(22,24)$. Registerlinked studies present increased risks of breast cancer in the nursing profession (95-101), but case-control studies that include information on reproductive factors or other potential confounders are in general negative (102-106).

Blind persons that may have lower sensitivity to light exposure have a decreased risk of breast cancer, and this occurrence is in line with a cumulative effect of light at night (and nightshift work). But this finding is not in line with the circadian disruption hypothesis because many visually impaired persons have free running cycles, and, furthermore, it is not clear whether blind persons secrete more melatonin than people who can see. Studies of light at night at home are supportive of a causal effect of nightshift work, but vulnerable to information bias, and thus have to be interpreted carefully.

Only two prospective studies of melatonin level and cancer risk have been conducted, and they present conflicting results. No data are currently available showing such an effect for instable melatonin rhythms.

\section{Previous literature reviews}

I identified 26 previous reviews or hypothesis papers about nightshift work, light at night, and the risk of cancer. The conclusions were generally in line with that of Megdal et al (3): "In summary, this meta-analysis suggests that shift work, including work as a flight attendant, increases the risk of breast cancer by $48 \%$ [p 2031]"; Hansen (45): “. . . there is so far relative consisting evidence that working non-day time may increase the breast cancer risk among females [p 534]"; and Davis et al (43): "Collectively these findings provide intriguing evidence that suggest working at night, or in occupations characterized by night shift work, may be associated with an increased risk of cancer [p 540]". Anthony Swerdlow (107) was more careful in his conclusion: "Hence, the possibility that shift work per se increases the risk of 
breast cancer cannot be dismissed, but on the other hand it remains possible that the apparent associations are due to confounding [p 18]". None of these reviews included the papers published in 2006 and 2007 by O'Leary et al (6), Kubo et al (7), Conlon et al (8), and Schwartzbaum et al (9) that are included in this review.

\section{Concluding remarks}

Five of the eight studies on breast cancer in women indicate increased risks. Three of the positive studies show significantly increased risks after 20-30 years of nightshift work. Hence there are indications of an effect of long-term nightshift work on breast cancer risk. Findings for shorter durations of nightshift work are inconsistent. There are several reasons for caution regarding the suggested long-term effect. The number of studies is small, the positive studies were all conducted for the same occupational group (nurses on nightshift), and the risk estimates are only moderately raised. These reasons make the results sensitive to bias, chance, and confounding, although I have not been able to pinpoint specific sources.

In conclusion, there is limited evidence for a causal association between nightshift work and breast cancer $(+)$, while there is insufficient evidence of a causal association for prostate cancer $(0)$, colon cancer $(0)$, and overall cancer (0). An expert working group convened by the International Agency for Research on Cancer came to the same conclusion at their meeting in October 2007, that there is limited evidence for the carcinogenicity of shift work that involves night work among humans (108).

Nightshift work is prevalent, the incidence of breast cancer is high, and an even slightly increased risk may carry a heavy burden for public health. A wealth of new studies on the physiological and pathophysiological effects of environmental lighting and circadian disruption are expected to provide path-breaking new insights (109). Still, further epidemiologic studies of nightshift work and the risk of breast cancer, as well as of other health effects, are needed. These studies should especially focus on reliable exposure data obtained on an individual level and include a wide range of occupational groups.

\section{Acknowledgments}

This study was funded by the Working Environment Research Fund and conducted at the initiative of the National Board of Industrial Injuries.

The author received valuable support from Johnni Hansen and Anders Ahlbom, who reviewed the manuscript together with the Scientific Committee of the
Danish Society for Occupational and Environmental Medicine, consisting of Staffan Skerfving, Johan Hviid Andersen, and Susanne Wulff Svendsen, which discussed the manuscript during a 1-day seminar on 23 August 2007.

\section{References}

1. Stevens RG. Electric power use and breast cancer: a hypothesis. Am J Epidemiol. 1987;125:556-61.

2. Stevens RG. Artificial lighting in the industrialized world: circadian disruption and breast cancer. Cancer Causes Control. 2006;17:501-7.

3. Megdal SP, Kroenke CH, Laden F, Pukkala E, Schernhammer ES. Night work and breast cancer risk: a systematic review and meta-analysis. Eur J Cancer. 2005;41:2023-32.

4. Taylor PJ, Pocock SJ. Mortality of shift and day workers 1956-68. Br J Ind Med. 1972;29:201-7.

5. Rafnsson V, Gunnarsdottir H. Mortality study of fertiliser manufacturers in Iceland. Br J Ind Med. 1990;47:721-5.

6. O'Leary ES, Schoenfeld ER, Stevens RG, Kabat GC, Henderson K, Grimson R, et al. Shift work, light at night, and breast cancer on Long Island, New York. Am J Epidemiol. 2006;164:358-66.

7. Kubo T, Ozasa K, Mikami K, Wakai K, Fujino Y, Watanabe $\mathrm{Y}$, et al. Prospective cohort study of the risk of prostate cancer among rotating-shift workers: findings from the Japan collaborative cohort study. Am J Epidemiol. 2006;164:549-55.

8. Conlon M, Lightfoot N, Kreiger N. Rotating shift work and risk of prostate cancer. Epidemiology. 2007;18:182-3.

9. Schwartzbaum J, Ahlbom A, Feychting M. Cohort study of cancer risk among male and female shift workers. Scand J Work Environ Health. 2007;33(5):336-43.

10. Ferlay J, Autier P, Boniol M, Heanue M, Colombet M, Boyle P. Estimates of the cancer incidence and mortality in Europe in 2006. Ann Oncol. 2007;18:581-92.

11. Madigan MP, Ziegler RG, Benichou J, Byrne C, Hoover RN. Proportion of breast cancer cases in the United States explained by well-established risk factors. J Natl Cancer Inst. 1995;87:1681-5.

12. Sasco AJ, Kaaks R, Little RE. Breast cancer: occurrence, risk factors and hormone metabolism. Expert Rev Anticancer Ther. 2003;3:546-62.

13. Yager JD, Davidson NE. Estrogen carcinogenesis in breast cancer. N Engl J Med. 2006;354:270-82.

14. Collaborative Group on Hormonal Factors in Breast Cancer. Breast cancer and hormonal contraceptives: collaborative reanalysis of individual data on 53297 women with breast cancer and 100239 women without breast cancer from 54 epidemiological studies. Lancet. 1996;347:1713-27.

15. Collaborative Group on Hormonal Factors in Breast Cancer. Breast cancer and hormone replacement therapy: collaborative reanalysis of data from 51 epidemiological studies of 52,705 women with breast cancer and 108,411 women without breast cancer. Lancet. 1997;350:1047-59.

16. Hamajima N, Hirose K, Tajima K, Rohan T, Calle EE, Heath $\mathrm{CW} \mathrm{Jr}$, et al. Alcohol, tobacco and breast cancer-collaborative reanalysis of individual data from 53 epidemiological studies, including 58,515 women with breast cancer and 95,067 women without the disease. Br J Cancer. 2002;87:1234-45.

17. Krebs EE, Taylor BC, Cauley JA, Stone KL, Bowman PJ, 
Ensrud KE. Measures of adiposity and risk of breast cancer in older postmenopausal women. J Am Geriatr Soc. 2006;54:639.

18. European Foundation for the Improvement of living and Working Conditions. Fourth European Working Conditions Survey. Luxembourg: Office for Official Publications of the European Communities; 2007.

19. European Foundation for the Improvement of living and Working Conditions. Third European survey on working conditions 2000. Luxembourg: Office for Official Publications of the European Communities; 2001.

20. Figueiro MG, Rea MS, Bullough JD. Does architectural lighting contribute to breast cancer? J Carcinog. 2006;5:20.

21. Bøggild H, Knutsson A. Shift work, risk factors and cardiovascular disease [review]. Scand J Work Environ Health. 1999;25(2):85-99.

22. Schernhammer ES, Laden F, Speizer FE, Willett WC, Hunter DJ, Kawachi I, et al. Rotating night shifts and risk of breast cancer in women participating in the nurses' health study. J Natl Cancer Inst. 2001;93:1563-8.

23. Schernhammer ES, Laden F, Speizer FE, Willett WC, Hunter DJ, Kawachi I, et al. Night-shift work and risk of colorectal cancer in the nurses' health study. J Natl Cancer Inst. 2003;95:825-8.

24. Schernhammer ES, Kroenke CH, Laden F, Hankinson SE. Night work and risk of breast cancer. Epidemiology. 2006;17:108-11.

25. Hansen J. Increased breast cancer risk among women who work predominantly at night. Epidemiology. 2001;12:74-7.

26. Ingre $\mathrm{M}, \mathrm{Akerstedt} \mathrm{T}$. Effect of accumulated night work during the working lifetime, on subjective health and sleep in monozygotic twins. J Sleep Res. 2004;13:45-8.

27. Knutsson A. Methodological aspects of shift-work research. Chronobiol Int. 2004;21:1037-47.

28. Härmä M. Workhours in relation to work stress, recovery and health [review]. Scand J Work Environ Health. 2006;32(6, special issue):502-14.

29. Hansen AM, Garde AH, Hansen J. Diurnal urinary 6-sulfatoxymelatonin levels among healthy Danish nurses during work and leisure time. Chronobiol Int. 2006;23:1203-15.

30. Borugian MJ, Gallagher RP, Friesen MC, Switzer TF, Aronson KJ. Twenty-four-hour light exposure and melatonin levels among shift workers. J Occup Environ Med. 2005;47:126875.

31. Schernhammer ES, Rosner B, Willett WC, Laden F, Colditz GA, Hankinson SE. Epidemiology of urinary melatonin in women and its relation to other hormones and night work. Cancer Epidemiol Biomarkers Prev. 2004;13:936-43.

32. Davis S, Mirick DK, Stevens RG. Night shift work, light at night, and risk of breast cancer. J Natl Cancer Inst. 2001;93:1557-62.

33. Tynes T, Hannevik M, Andersen A, Vistnes AI, Haldorsen T. Incidence of breast cancer in Norwegian female radio and telegraph operators. Cancer Causes Control. 1996;7:197-204.

34. Lie JA, Roessink J, Kjaerheim K. Breast cancer and night work among Norwegian nurses. Cancer Causes Control. 2006;17:39-44.

35. Anisimov VN. The light-dark regimen and cancer development. Neuro Endocrinol Lett. 2002;23 suppl 2:28-36.

36. Anisimov VN. The role of pineal gland in breast cancer development. Crit Rev Oncol Hematol. 2003;46:221-34.

37. Anisimov VN. Light pollution, reproductive function and cancer risk. Neuro Endocrinol Lett. 2006;27:35-52.

38. Anisimov VN, Vinogradova IA. Light-dark conditions, mela- tonin and risk of cancer. Vopr Onkol. 2006;52:491-8.

39. Blask DE, Dauchy RT, Sauer LA. Putting cancer to sleep at night: the neuroendocrine/circadian melatonin signal. Endocrine. 2005;27:179-88.

40. Bullough JD, Rea MS, Figueiro MG. Of mice and women: light as a circadian stimulus in breast cancer research. Cancer Causes Control. 2006; 17:375-83.

41. Cornelissen G, Halberg F. Chronobiologic response modifiers and breast cancer development: classical background and chronobiologic tasks remaining. In Vivo. 1992;6:387-402.

42. Coyle YM. The effect of environment on breast cancer risk. Breast Cancer Res Treat. 2004;84:273-88.

43. Davis S, Mirick DK. Circadian disruption, shift work and the risk of cancer: a summary of the evidence and studies in Seattle. Cancer Causes Control. 2006;17:539-45.

44. Feychting M, Ahlbom A. Kan ljusexponering påverka risken för bröstcancer [Can light exposure affect the risk of breast cancer?: female flight attendants and shift-workers probably the most vulnerable groups]. Läkartidningen. 2001;98:4316-8.

45. Hansen J. Risk of breast cancer after night- and shift work: current evidence and ongoing studies in Denmark. Cancer Causes Control. 2006;17:531-7.

46. Haus E, Smolensky M. Biological clocks and shift work: circadian dysregulation and potential long-term effects. Cancer Causes Control. 2006;17:489-500.

47. Jasser SA, Blask DE, Brainard GC. Light during darkness and cancer: relationships in circadian photoreception and tumor biology. Cancer Causes Control. 2006;17:515-23.

48. Kenny C. Breast cancer: night shifts put nurses at risk. Nurs Times. 2001;97:9.

49. Kheifets LI, Matkin CC. Industrialization, electromagnetic fields, and breast cancer risk. Environ Health Perspect. 1999;107 suppl 1:145-54.

50. Patel D. Shift work, light at night and risk of breast cancer. Occup Med (Lond). 2006;56:433.

51. Pauley SM. Lighting for the human circadian clock: recent research indicates that lighting has become a public health issue. Med Hypotheses. 2004;63:588-96.

52. Schernhammer E, Schulmeister K. Light at night and cancer risk. Photochem Photobiol. 2004;79:316-8.

53. Schernhammer ES, Schulmeister K. Melatonin and cancer risk: does light at night compromise physiologic cancer protection by lowering serum melatonin levels? Br J Cancer. 2004;90:941-3.

54. Stevens RG. Lighting during the day and night: possible impact on risk of breast cancer. Neuro Endocrinol Lett. 2002;23 suppl 2:57-60.

55. Stevens RG. Circadian disruption and breast cancer: from melatonin to clock genes. Epidemiology. 2005;16:254-8.

56. Stevens RG, Rea MS. Light in the built environment: potential role of circadian disruption in endocrine disruption and breast cancer. Cancer Causes Control. 2001;12:279-87.

57. Bonde JP, Andersen JH, Frost P, Kaergaard A, Kolstad HA, Thulstrup AM. Helbredsundersøgelse ved natarbejde [Health examinations in connection with night work]. Ugeskr Laeger. 2007;169:2005-7.

58. Frentzel-Beyme R. The melatonin hypothesis: a matter of method. Environ Health Perspect. 2002;110:A72-A73.

59. Hrushesky WJ, Blask DE. Re: Melatonin and breast cancer: a prospective study. J Natl Cancer Inst. 2004;96:888-9.

60. Kerenyi N. Re: Night shift work, light at night, and risk of breast cancer. J Natl Cancer Inst. 2002;94:531-2.

61. Porock D, Gentry J. Re: Night shift work, light at night, and risk of breast cancer. J Natl Cancer Inst. 2002;94:530-1. 
62. Spiegel D, Sephton S. Re: Night shift work, light at night, and risk of breast cancer. J Natl Cancer Inst. 2002;94:530-3.

63. Tarone RE. Circadian disruption and breast cancer. Epidemiology. 2005;16:710-1.

64. Hansen J. Light at night, shiftwork, and breast cancer risk. J Natl Cancer Inst. 2001;93:1513-5.

65. Poole $\mathrm{C}$. The darkness at the end of the tunnel: summary and evaluation of an international symposium on light, endocrine systems and cancer. Neuro Endocrinol Lett. 2002;23 suppl 2:71-8.

66. Roenneberg T, Lucas RJ. Light, endocrine systems, and cancer-a view from circadian biologists. Neuro Endocrinol Lett. 2002;23 suppl 2:82-3.

67. Night shifts and colorectal cancer risk. Mayo Clin Health Lett. 2005;23:4.

68. Kojo K, Pukkala E, Auvinen A. Breast cancer risk among Finnish cabin attendants: a nested case-control study. Occup Environ Med. 2005;62:488-93.

69. Rafnsson V, Hrafnkelsson J, Tulinius H. Incidence of cancer among commercial airline pilots. Occup Environ Med. 2000;57:175-9.

70. Rafnsson V, Tulinius H, Jonasson JG, Hrafnkelsson J. Risk of breast cancer in female flight attendants: a population-based study (Iceland). Cancer Causes Control. 2001;12:95-101.

71. Gurwitz D. Flight attendants, breast cancer, and melatonin. Lancet. 1998;352:1389-90.

72. Mawson AR. Breast cancer in female flight attendants. Lancet. 1998;352:626.

73. Radon K, Aberl S, Nowak D, Volkenandt M, Przybilla BJ, Siebeck $\mathrm{M}$, et al. Incidence of cancer among commercial airline pilots. Occup Environ Med. 2000;57:843.

74. Cook MR, Graham C, Kavet R, Stevens RG, Davis S, Kheifets L. Morning urinary assessment of nocturnal melatonin secretion in older women. J Pineal Res. 2000;28:41-7.

75. Davis S, Kaune WT, Mirick DK, Chen C, Stevens RG. Residential magnetic fields, light-at-night, and nocturnal urinary 6sulfatoxymelatonin concentration in women. Am J Epidemiol. 2001;154:591-600.

76. Schernhammer ES, Hankinson SE. Urinary melatonin levels and breast cancer risk. J Natl Cancer Inst. 2005;20;97:1084-7.

77. Schernhammer ES, Kroenke CH, Dowsett M, Folkerd E, Hankinson SE. Urinary 6-sulfatoxymelatonin levels and their correlations with lifestyle factors and steroid hormone levels. J Pineal Res. 2006;40:116-24.

78. Stevens RG, Schernhammer E. Epidemiology of urinary melatonin in women and its relation to other hormones and night work. Cancer Epidemiol Biomarkers Prev. 2005;14:551.

79. Zhu Y, Zheng T, Stevens RG, Zhang Y, Boyle P. Does “clock" matter in prostate cancer? Cancer Epidemiol Biomarkers Prev. 2006;15:3-5.

80. Gammon MD, Neugut AI, Santella RM, Teitelbaum SL, Britton JA, Terry MB, et al. The Long Island Breast Cancer Study Project: description of a multi-institutional collaboration to identify environmental risk factors for breast cancer. Breast Cancer Res Treat. 2002;74:235-54.

81. Pukkala E, Aspholm R, Auvinen A, Eliasch H, Gundestrup M, Haldorsen $\mathrm{T}$, et al. Incidence of cancer among Nordic airline pilots over five decades: occupational cohort study. BMJ. 2002;325:567.

82. Zeeb H, Blettner M, Langner I, Hammer GP, Ballard TJ, Santaquilani M, et al. Mortality from cancer and other causes among airline cabin attendants in Europe: a collaborative cohort study in eight countries. Am J Epidemiol. 2003;158:35-46.

83. Rafnsson V, Sulem P, Tulinius H, Hrafnkelsson J. Breast cancer risk in airline cabin attendants: a nested case-control study in Iceland. Occup Environ Med. 2003;60:807-9.

84. Pukkala E, Ojamo M, Rudanko SL, Stevens RG, Verkasalo PK Does incidence of breast cancer and prostate cancer decrease with increasing degree of visual impairment. Cancer Causes Control. 2006;17:573-6.

85. Sack RL, Lewy AJ, Blood ML, Keith LD, Nakagawa H. Circadian rhythm abnormalities in totally blind people: incidence and clinical significance. J Clin Endocrinol Metab. 1992;75:127-34.

86. Lockley SW, Skene DJ, Arendt J, Tabandeh H, Bird AC, Defrance R. Relationship between melatonin rhythms and visual loss in the blind. J Clin Endocrinol Metab. 1997;82:3763-70.

87. Klerman EB, Zeitzer JM, Duffy JF, Khalsa SB, Czeisler CA. Absence of an increase in the duration of the circadian melatonin secretory episode in totally blind human subjects. J Clin Endocrinol Metab. 2001;86:3166-70.

88. Travis RC, Allen DS, Fentiman IS, Key TJ. Melatonin and breast cancer: a prospective study. J Natl Cancer Inst 2004;96:475-82.

89. Blask DE, Brainard GC, Dauchy RT, Hanifin JP, Davidson LK, Krause JA, et al. Melatonin-depleted blood from premenopausal women exposed to light at night stimulates growth of human breast cancer xenografts in nude rats. Cancer Res. 2005;65:11174-84.

90. Sanchez-Barcelo EJ, Cos S, Fernandez R, Mediavilla MD Melatonin and mammary cancer: a short review. Endocr Relat Cancer. 2003;10:153-9.

91. Anderson LE, Morris JE, Sasser LB, Stevens RG. Effect of constant light on DMBA mammary tumorigenesis in rats. Cancer Lett. 2000;148:121-6.

92. Barnes RG, Deacon SJ, Forbes MJ, Arendt J. Adaptation of the 6-sulphatoxymelatonin rhythm in shiftworkers on offshore oil installations during a 2-week 12-h night shift. Neurosci Lett. 1998;241:9-12.

93. Margolese RG, Hortobagyi GN, Buchholz TA. Neoplasms of the breast. In: Kufe DW, Pollock RE, Weicheslbaum RR, Bast RC, Gansler TS, Holland JF, et al, editors. Cancer medicine. Hamilton (Ontario): BC Decker Inc; 2003:1879-970.

94. Colditz GA. The nurses' health study: a cohort of US women followed since 1976. J Am Med Womens Assoc. 1995;50:40-4.

95. Gunnarsdottir H, Rafnsson V. Cancer incidence among Icelandic nurses. J Occup Environ Med. 1995;37:307-12.

96. Morton WE. Major differences in breast cancer risks among occupations. J Occup Environ Med. 1995;37:328-35.

97. Petralia SA, Chow WH, McLaughlin J, Jin F, Gao YT, Dosemeci M. Occupational risk factors for breast cancer among women in Shanghai. Am J Ind Med. 1998;34:477-83.

98. Peipins LA, Burnett C, Alterman T, Lalich N. Mortality patterns among female nurses: a 27-state study, 1984 through 1990. Am J Public Health. 1997;87:1539-43.

99. Andersen A, Barlow L, Engeland A, Kjærheim K, Lynge E, Pukkala E. Work-related cancer in the Nordic countries. Scand J Work Environ Health. 1999;25 suppl 2:1-116.

100. Lie JA, Andersen A, Kjærheim K. Cancer risk among 43000 Norwegian nurses. Scand J Work Environ Health. 2007;33(1):66-73.

101. MacArthur AC, Le ND, Abanto ZU, Gallagher RP. Occupational female breast and reproductive cancer mortality in British Columbia, Canada, 1950-94. Occup Med (Lond). 2007;57:246-53.

102. Habel LA, Stanford JL, Vaughan TL, Rossing MA, Voigt LF, Weiss NS, et al. Occupation and breast cancer risk in middleaged women. J Occup Environ Med. 1995;37:349-56. 
103. Coogan PF, Clapp RW, Newcomb PA, Mittendorf R, Bogdan $\mathrm{G}$, Baron JA, et al. Variation in female breast cancer risk by occupation. Am J Ind Med. 1996;30:430-7.

104. Band PR, Le ND, Fang R, Deschamps M, Gallagher RP, Yang P. Identification of occupational cancer risks in British Columbia: a population-based case-control study of 995 incident breast cancer cases by menopausal status, controlling for confounding factors. J Occup Environ Med. 2000;42:284-310.

105. Lie JA, Kjaerheim K. Cancer risk among female nurses: a literature review. Eur J Cancer Prev. 2003;12:517-26.

106. Petralia SA, Vena JE, Freudenheim JL, Michalek A, Goldberg MS, Blair A, et al. Risk of premenopausal breast cancer and patterns of established breast cancer risk factors among teach- ers and nurses. Am J Ind Med. 1999;35:137-41.

107. Swerdlow, A. Shift work and breast cancer; a critical review of the epidemiological evidence. Norwich (United Kingdom): Health and Safety Executive; 2003.

108. Straif K, Baan R, Grosse Y, Secretan B, El Ghissassi F, Bouvard V, et al. Carcinogenicity of shift-work, painting, and firefighting. Lancet Oncol. 2007;8:1065-6.

109. Stevens RG, Blask DE, Brainard GC, Hansen J, Lockley SW, Provencio I, et al. Meeting report: the role of environmental lighting and circadian disruption in cancer and other diseases. Environ Health Perspect. 2007;115:1357-62.

Received for publication: 27 September 2007

\section{Appendix 1}

\section{Degree of evidence of a causal association between exposure to a specific risk factor and a specific out- come according to criteria of the Scientific Committee of the Danish Society of Occupational and Environ- mental Medicine}

The following categories are used.

+++ strong evidence of a causal association

++ moderate evidence of a causal association

$+\quad$ limited evidence of a causal association
0 insufficient evidence of a causal association

- evidence suggesting lack of a causal association

\section{Description of categories}

Strong evidence of a causal association (+++). A causal relationship is very likely. A positive relationship between exposure to the risk factor and the outcome has been observed in several epidemiologic studies. It can be ruled out with reasonable confidence that this relationship is explained by chance, bias, or confounding.

Moderate evidence of a causal association (++). A causal relationship is likely. A positive relationship between exposure to the risk factor and the outcome has been observed in several epidemiologic studies. It cannot be ruled out with reasonable confidence that this relationship can be explained by chance, bias, or confounding, although this is not a very likely explanation.

Limited evidence of a causal association (+). A causal relationship is possible. A positive relationship between exposure to the risk factor and the outcome has been observed in several epidemiologic studies. It is not unlikely that this relationship can be explained by chance, bias, or confounding.

Insufficient evidence of a causal association (0). The available studies are of insufficient quality, consistency, or statistical power to permit a conclusion regarding the presence or absence of a causal association.

Evidence suggesting a lack of a causal association (-). Several studies of sufficient quality, consistency, and statistical power indicate that the specific risk factor is not causally related to the specific outcome.

\section{Comments}

The classification does not include a category for which a causal relation is considered as established beyond any doubt.

The key criterion is the epidemiologic evidence.

The likelihood that chance, bias, and confounding may explain observed associations are criteria that encompass criteria such as consistency, number of "high quality" studies, types of design, and the like.

Biological plausibility and contributory information may add to the evidence of a causal association. 